\title{
Separating Turbofan Engine Noise Sources Using Auto- and Cross Spectra from Four Microphones
}

\author{
Jeffrey Hilton Miles* \\ NASA John H. Glenn Research Center at Lewis Field, \\ Cleveland, Ohio 44135 \\ DOI: $\underline{10.2514 / 1.25177}$
}

\begin{abstract}
The study of core noise from turbofan engines has become more important as noise from other sources such as the fan and jet were reduced. A multiple-microphone and acoustic-source modeling method to separate correlated and uncorrelated sources is discussed. The auto- and cross spectra in the frequency range below $1000 \mathrm{~Hz}$ are fitted with a noise propagation model based on a source couplet consisting of a single incoherent monopole source with a single coherent monopole source or a source triplet consisting of a single incoherent monopole source with two coherent monopole point sources. Examples are presented using data from a Pratt \& Whitney PW4098 turbofan engine. The method separates the low-frequency jet noise from the core noise at the nozzle exit. It is shown that at low power settings, the core noise is a major contributor to the noise. Even at higher power settings, it can be more important than jet noise. However, at low frequencies, uncorrelated broadband noise and jet noise become the important factors as the engine power setting is increased.
\end{abstract}

$A$

$B$

$B_{e}$

$c_{o}$

$\operatorname{conp} 2 n 50$

E

$F[]$

$f$

$f_{c}$

$G_{x y}(f)$

$G_{x x}(f)$

$j$

$k$

$L_{y}$

$M W$

$N$

$N P$

$n_{d}$

$n_{o}$

$P_{I}$

\section{Nomenclature}

$=$ amplitude of a coherent monopole point source $A$

$=$ amplitude of a coherent monopole point source $B$

$=$ resolution bandwidth, $B_{e}=1 / T_{d}=$ $r_{s} / N P=11.71875 \mathrm{~Hz}$

$=$ speed of sound, $\mathrm{m} / \mathrm{s}$

$=$ function providing solution space boundary on $\mathrm{SPL}_{N}$

$=$ expected value

$=$ two-sided Fourier transform operator

$=$ frequency

$=$ upper frequency limit, $f_{c}=1 /(2 \Delta t)=r_{s} / 2$, $24,000 \mathrm{~Hz}$

$=$ cross power spectral density function defined for nonnegative frequencies only (one-sided)

$=$ auto power spectral density function defined for nonnegative frequencies only (one-sided)

$=$ imaginary unit, $\sqrt{-1}$

$=$ wave number, $2 \pi f / c_{o}, \mathrm{~m}^{-1}$

$=$ number of frequencies, $f_{c} / \Delta f=N / 2$ (2048)

$=$ molecular weight of air, $28.97, \mathrm{~kg} / \mathrm{k} \mathrm{mol}$

$=$ amplitude of uncorrelated monopole point noise source, $\mathrm{N}$

$=$ segment length, number of data points per segment (4096)

$=$ number of disjoint (independent) segments, $n_{d}=B_{e} t_{\mathrm{total}}=234$

$=$ number of overlapped segments/blocks

$=$ probability confidence interval, $\%$

Presented as Paper 2580 at the 12th AIAA/CEAS Aeroacoustics Conference, Cambridge, MA, 8-10 May 2006; received 15 May 2006; revision received 31 May 2007; accepted for publication 16 June 2007. This material is declared a work of the U.S. Government and is not subject to copyright protection in the United States. Copies of this paper may be made for personal or internal use, on condition that the copier pay the $\$ 10.00$ percopy fee to the Copyright Clearance Center, Inc., 222 Rosewood Drive, Danvers, MA 01923; include the code 0001-1452/08 $\$ 10.00$ in correspondence with the CCC.

*Aerospace Engineer, Acoustics Branch, 21000 Brookpark Road. Senior Member AIAA.

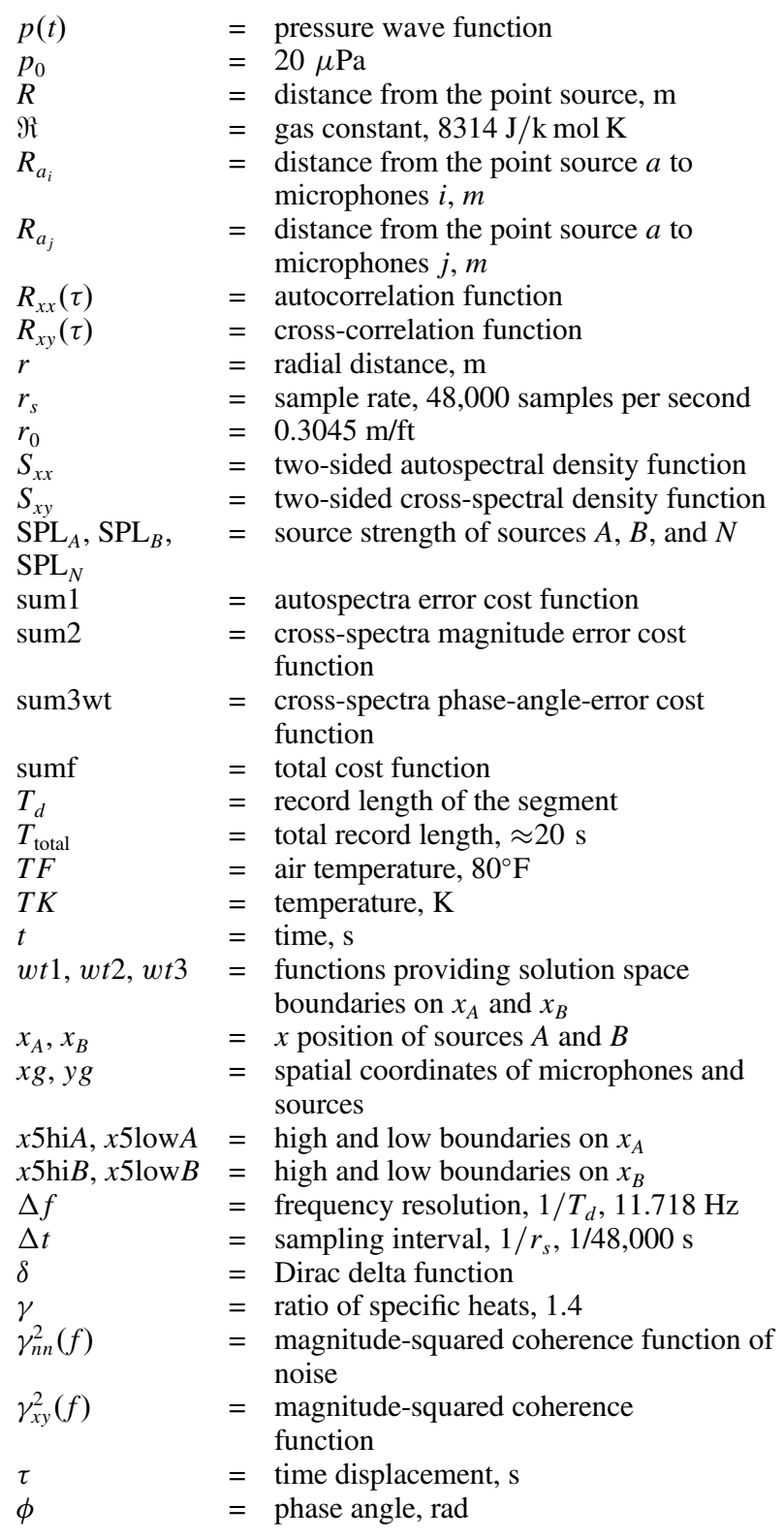




\section{Subscripts}

$o$

$x$

$y$
$=$ some arbitrary specific value

$=\operatorname{signal} x$

$=$ signal $y$

\section{Introduction}

$\mathbf{U}$ NDERSTANDING turbofan engine noise is a key priority of NASA. Consequently, diagnostic procedures to identify dominant sources and changes in dominant sources are being developed. This paper discusses a diagnostic procedure that separates correlated far-field turbofan noise from uncorrelated farfield turbofan noise using a multiple-microphone method and pointsource acoustic models. The auto- and cross spectra in the frequency range below $1000 \mathrm{~Hz}$ are fitted with a noise propagation model based on a source couplet consisting of a single incoherent source with a single coherent source or a source triplet consisting of a single incoherent source with two coherent point sources. Two noise signals are coherent if they can be aligned with one another so that the coherence calculated using a periodogram averaging method is greater than the coherence of two random signals using the same periodogram averaging method [1]. As fan noise and jet noise from turbofan engines are reduced, the issue of core-noise reduction becomes more important. Although core noise may be reduced by acoustic liners, the issue of measuring the amount of reduction becomes increasingly important. The proposed scheme separates coherent noise from random jet noise and should enable the measurement of the effectiveness of core-noise reduction liners. The scheme was developed as part of a research program to study core noise from a Pratt \& Whitney PW4098 turbofan engine (Miles [1-3] .)

Procedures using coherence-based techniques have been developed for extracting acoustic signals buried in noise. The coherent output power method for noise-source identification is discussed by Bendat and Piersol [4-6]. The application of this technique that is of interest is the use of coherent output power spectra to separate and identify correlated combustion noise in farfield measurements of turbofan engine noise. Karchmer [7] and Karchmer et al. [8], use the coherence function calculated from internal microphone measurements of fluctuating pressures in the combustor and far-field acoustic pressures to determine the correlated combustion noise of a YF102 turbofan engine at far-field locations by calculating the coherent output power spectrum.

The three-signal coherence technique was developed by Chung [9,10] for flow noise rejection. A similar technique was developed and used by Krejsa [11]. The three-signal coherence technique was used by Shivashankara [12] to study core noise in a Pratt \& Whitney JT9D. It was used by Hsu and Ahuja [13] to separate ejector internal mixing noise from far-field measurements and by Stoker et al. [14] to separate wind-tunnel background noise and wind noise from automobile interior noise measurements. It was used by Michalke et al. [15] to study sound in a circular duct with mean flow. The method is also discussed by Minami and Ahuja [16].

The inherent coherent properties of jet and core noise have been used in several source-separation procedures. The radiated field of jet noise has a low coherence when measured at two widely separated points. Parthasarathy et al. [17] attributed this to the fact that the jetnoise sources are in motion so that the source frequencies undergo large Doppler shifts as the noise is radiated to the far-field. Core noise has a high coherence when measured at two widely separated points. This is attributed to the fact that the frequencies of the radiated core noise are preserved unchanged in the far field (Parthasarathy et al. [17]). Consequently, three-signal coherence technique is especially useful to study turbofan core noise using widely spaced microphones.

A method of separating jet noise and core noise using autocorrelations and cross correlations was developed by Parthasarathy et al. [17]. The model presented assumes that the

microphones are located on the arc of a circle about a single source having a known position. This is a couplet source, which produces a core-noise sound radiation field that is correlated over wide microphone spacing and a jet-noise sound radiation field that is not correlated over wide microphone spacing. Because the microphones are on the same arc, the model cross correlations calculated have zero propagation time delay. In addition, a model for the jet-noise directivity is used. The resulting system of three unknowns and three equations can be solved exactly. In applying this method to data from a small jet, the source location is known and a correction to the amplitude correlation measurements is made so that the microphones are located on the arc of a circle about a source having a known position. In applying this method to measured data, I assume the cross correlations used by Parthasarathy et al. were also time-shifted to remove the propagation time delay. An extension of the method to obtain spectral information is also discussed using nonzero values of propagation time delay. The phase-angle shift due to propagation time delay is not discussed. Again, this seems to indicate that the propagation time delay is being set to zero. The method calculates the jet-noise spectrum, the core-noise spectrum, and the directivity ratio of the core noise at a particular angle as a function of frequency from measured auto- and cross spectra. The method was applied to experimental data obtained in a small-scale facility. The spectral formulation of this method was used by Tesson $[18,19]$ to study jet noise and core noise from the static test of a small gas turbine engine in an anechoic chamber.

A method to achieve separation of two different correlated noise sources from far-field measurements contaminated by extraneous jet noise using multiple microphones was developed by Minami and Ahuja [16]. The equations discussed use measured autospectra and cross spectra. The model assumes that the source noise can be represented by a triplet source consisting of a correlated noise source $A$, correlated noise source $B$, and an uncorrelated noise source. In this analysis by Minami and Ahuja, it is assumed that all the sources are at a single triplet location; however, in the formulation presented, no source location or microphone location information is used. The fivemicrophone method described involves solving a set of 55 equations for 55 unknowns at each frequency of interest. At each microphone location, the spectrum of correlated noise source $A$, correlated noise source $B$, and an uncorrelated noise source is obtained as a function of frequency. The method was validated using analytical simulations.

Beamforming and phased-array methods used in acoustic testing require similar measurements and use a similar source description [20-26]. However, they use more than four microphones and are based on a fixed geometry, whereas the procedure described herein uses a floating geometry. A phased-array procedure using a linear array of 14 microphones was used to study low-frequency noise from a Rolls-Royce/SNECMA Olympus engine by Flynn and Kinns [27] and Billingsley and Kinns [28]. Concurrently, the polar correlation technique was developed by Fisher et al. [29] and used to study Rolls-Royce Viper 601 engine noise and Rolls-Royce RB211 engine noise.

Source characterization by correlation techniques used by Blake and Lynch [30] to study trailing-edge noise and fan noise require similar measurements. A procedure for source characterization using a closely spaced pair of microphones in the far field was developed by Kinns [31].

As pointed out by Williams [32] in a discussion of antisound, there is no unique specification for the origin of a given sound field. One may only think in terms of an equivalent source region that shares the measurable far-field properties of the real sources. As he states, "There is no difference between the sound of a naturally occurring phase-related multiple-source system and that created artificially with control (electronic and acoustic apparatus) but the latter can be changed and adapted at the 'flick of a switch' as it were." The sourceseparation method described herein uses this concept. The particular source models described were chosen for convenience and have a feasible analogy. The chosen mathematical models permit the creation of the correct directivity and phase relationships between the microphone signals as a function of frequency. 
The method presented herein also models the auto- and crossspectrum measurements made with multiple microphones. Rather than use a single mathematical-equation-based model, the method uses a sequence of models. Each model consists of a collection of one or more point-source solutions to a three-dimensional acoustic wave equation in spherical coordinate form. Each element of the sequence of models is based on propagation from a collection of point-source sound radiators on the engine axis that can have arbitrary positions on the engine axis. Consequently, the microphones need not be on a circular arc at the same distance from the source. In addition, for each source, the directivity is the same in each direction for each source. The method tests the measured data at each frequency against various models. The first model in the sequence assumes an incoherent source of strength $\operatorname{SPL}_{N}(f)$ at the location $x_{N}=0.0$ and $y_{N}=0.0$. The second model in the sequence assumes that the source is a doublet consisting of an incoherent source of strength $\operatorname{SPL}_{N}(f)$ at the location $x_{N}=0.0$ and $y_{N}=0.0$ and an additional coherent source of strength $\mathrm{SPL}_{A}(f)$ at location $x_{A}(f)$ with $y_{A}=0.0$. The third model in the sequence assumes that the data are the result of a source triplet consisting of an incoherent source of strength $\operatorname{SPL}_{N}(f)$ at the location $x_{N}=0.0$ and $y_{N}=0.0$, a coherent source at location $x_{A}(f)$ with $y_{A}=0.0$ of strength $\operatorname{SPL}_{A}(f)$, and an additional coherent source at location $x_{B}(f)$ with $y_{B}=0.0$ of strength $\operatorname{SPL}_{B}(f)$.

For each model in the sequence, the appropriate parameters were adjusted to give the best fit to the data at each frequency. The results presented here are those obtained by the method when one assumes that the engine noise source can be modeled with a source doublet or triplet.

One part of a source doublet and triplet consists of an incoherent source of strength $\operatorname{SPL}_{N}(f)$ at the location $x_{N}=0.0$ and $y_{N}=0.0$. The source doublet has an additional coherent source of strength $\mathrm{SPL}_{A}(f)$ at location $x_{A}(f)$ with $y_{A}=0.0$. The source triplet has two additional coherent sources at location $x_{A}(f)$ with $y_{A}=0.0$ and $x_{B}(f)$ with $y_{B}=0.0$. As part of the procedure, a comparison of results obtained with the use of a source doublet with the results obtained using a source triplet is made. A source position is assumed to have a $y$ coordinate of zero and the $x$ coordinate along the engine axis is found as a function of frequency. This is done because lowfrequency noise can be radiated to the far field from the inlet, the engine case, the exhaust nozzle, and the jet. To solve the resulting acoustic equations for a small set of parameters, a solution method that provides an optimum solution in a least-squares sense without derivatives was used. The method is set up to do a least-squares curve fit to match the autospectra and the cross-spectra magnitude and phase measurements between all microphones. The method assumes the existence of one or more point sources that can produce the same measurements. The problem then becomes one of finding the appropriate point sources. The current strategy is to have the curve fit adjust the amplitude and $x$ location of the sources that determine the cross spectrum at each microphone and the amplitude of a randomnoise source. For the single coherent source case, three parameters are found at each frequency. When a model with two coherent sources is used, one has five parameters to determine at each frequency. Examples are presented calculated with a fourmicrophone array using data from a Pratt \& Whitney PW4098 turbofan engine.

\section{Analysis Method}

The core noise is assumed to be propagating in acoustic modes in the turbofan engine. The coherent acoustic energy leaves the nozzle and travels through the turbofan engine shear layer to a ground microphone. Additional acoustic energy from the jet and from random scattering reaches the same microphone. In this test program, four microphones at $150 \mathrm{ft}$ and angular position of 100,110,120, and $130 \mathrm{deg}$ measured from the inlet were used. Consequently, the measurements available at each test condition are four soundpressure spectrums and six sound-pressure cross spectrums, each consisting of a magnitude and a phase angle. These 16 measurements are available as a function of frequency. Four real variable and six complex variable acoustic equations relate the measurements and the model parameters. The angular separation of at least 10 deg means that the jet noise measured at any microphone can be assumed to be incoherent above some frequency with the jet noise at any another microphone.

The basic procedure is to assume that some combination of coherent sources and an incoherent source will produce the measured auto- and cross spectra. Coherent sources are assumed to be on the turbofan engine centerline at some $x(f)$ position and with $y=0$. The incoherent source is assumed to be at $x=0$ and $y=0$. Using the results in Appendix A, in which the one-sided spectrum functions for acoustic signals from a point source are derived, the acoustic model equations shown in Appendices $\underline{\mathrm{B}}, \underline{\mathrm{C}}, \underline{\mathrm{D}}$, and $\underline{\mathrm{E}}$ are defined. The following models were considered:

1) The two-parameter model has a coherent source of magnitude $A$ at $x=x_{a}$ and $y=0$ (Appendix B).

2) The three-parameter model has a coherent source of magnitude $A$ at $x=x_{a}$ and $y=0$ (Appendix $\underline{\mathrm{C}}$ ) and an incoherent source of magnitude $N$ at $x=0$ and $y=0$.

3 ) The four-parameter model has a coherent source of magnitude $A$ at $x=x_{a}$ and $y=0$ and a coherent source of magnitude $B$ at $x=x_{b}$ and $y=0$ (Appendix D).

4) The five-parameter model has a coherent source of magnitude $A$ at $x=x_{a}$ and $y=0$ and a coherent source of magnitude $B$ at $x=x_{b}$ and $y=0$ with an incoherent source of magnitude $N$ at $x=0$ and $y=0$ (Appendix E).

Consequently, the number of equations greatly exceeds the number of model parameters used in the study discussed herein. Note that the radiation pattern assumed for these models is one that radiates equally in all directions. These are monopole sources.

The measured autospectra and cross spectra required as input to solve the acoustic model equations is experimentally determined and subject to nominal experimental error and statistical uncertainties. Also, the measured autospectrum includes random noise in addition to coherent signals from propagating waves. Consequently, a solution method that provides an optimum solution in a least-squares sense without derivatives was used. Algorithms for minimization of a cost function without derivatives are discussed by Brent [33]. The search technique used in this study is described by Powell [34], and a Fortran computer code for this algorithm is given by Shapiro and Goldstein [35] and Kuester and Mize [36]. The code used was a modified version of the one in Shapiro and Goldstein [35], which was updated to be in a Fortran-90 style.

A typical cost function is discussed in Appendix F. The cost function serves three purposes. In addition to providing a function defining the solution, the cost function is also used to break the symmetry for the case in which the solution has two coherent sources. A plot of the locations $x_{A}$ and $x_{B}$ can be confusing for this case because the coherent monopole sources are symmetric. Consequently, at a particular frequency, interchanging positions and magnitudes does not change the answer. However, the plots of the locations $x_{A}$ and $x_{B}$ can look strange. To explicitly break the symmetry, a penalty function is used to force the condition $x_{B}<x_{A}$.

The third function of the cost function is to confine the solution to a reasonable domain. After looking at the results of many unconstrained searches, two search regions were selected based on frequency. For $f<164 \mathrm{~Hz}$, we use $11<x_{A}<100$ and $-25<x_{B}<100$. However, for $f>164 \mathrm{~Hz}$, we use $11<x_{A}<25$ and $-25<x_{B}<11$ (where the locations are in units of feet). The resulting penalty functions prevent convergence to a solution in which $x_{A}=3000$.

\section{Experimental Setup}

To demonstrate the usefulness of the procedure for separating correlated and uncorrelated noise sources, measurements made in the far field of a Pratt \& Whitney PW4098 turbofan engine will be used. The measurements were made in a study of aircraft turbofan engine core noise conducted as part of the NASA Engine Validation of Noise Reduction Concepts (EVNRC) program.

The spectral estimate parameters are shown in Table 1 . The signal processing algorithms used were written in Fortran. They are based 
Table 1 Spectral estimate parameters

\begin{tabular}{lc}
\hline \hline Parameter & Value \\
\hline Segment length (data points per segment), $N P$ & 4096 \\
Sample rate $r$, samples per second & 48,000 \\
Segment length, $T_{d}=N P / r, \mathrm{~s}$ & 0.08533 \\
Sampling interval, $\Delta t=1 / r, \mathrm{~s}$ & $2.0833 \times 10^{-5}$ \\
Frequency step, $\Delta f=1 / T_{d}, \mathrm{~Hz}$ & 11.718 \\
Upper frequency limit, $f_{c}=1 / 2 \Delta t=r / 2, \mathrm{~Hz}$ & 24,000 \\
Number of frequencies, $L y=f_{c} / \Delta f=N p / 2$ & 2048 \\
Time delay, $\tau=6323 / 48,000 \mathrm{~s}$ & 0.1317 \\
Number of independent samples & 234 \\
Overlap & 0.50 \\
Sample length, $\mathrm{s}$ & 20 \\
\hline \hline
\end{tabular}

on subprogram modules developed by Stearns and David [37]. In the calculations, the segments were overlapped by $50 \%$. Figure 1 shows the angular placement of the far-field microphones on a $150-\overline{f t}$ radius. This analysis uses the microphones at 100, 110, 120, and $130 \mathrm{deg}$ measured from the inlet. The coordinate system used herein has the $x$ axis along the engine centerline, increasing to the right. The $y$ axis is perpendicular and increases toward the top of the plot. The center $(x=0$ and $y=0)$ is at the engine center.

In calculating the cross spectra between the microphones, no time delay is used. Consequently, the cross spectra between the microphones have a phase shift related to the time a wave front from a particular source reaches each microphone.

\section{A. Cross-Spectra Validity}

\section{Results}

The signals from each pair of microphones used to calculate the cross spectra only produce valid measurements if the coherence is greater than some threshold coherence. The coherence function is given by

$$
\gamma_{\theta_{1} \theta_{2}}^{2}(f)=\frac{\left|G_{\theta_{1} \theta_{2}}(f)\right|^{2}}{G_{\theta_{1} \theta_{1}}(f) G_{\theta_{2} \theta_{2}}(f)}
$$

The measured coherence calculated using segments overlapped by $50 \%$ is given by

$$
\hat{\gamma}_{\theta_{1} \theta_{2}}^{2}(f)=\frac{\left|\sum_{i=1}^{n_{o}} X_{\theta_{1}}^{*}(i, f) X_{\theta_{2}}(i, f)\right|^{2}}{\sum_{i=1}^{n_{o}}\left|X_{\theta_{1}}(i, f)\right|^{2} \sum_{i=1}^{n_{o}}\left|X_{\theta_{2}}(i, f)\right|^{2}}
$$

In Miles [1], comparisons were made of a coherence threshold based on aligned and unaligned coherence and one based on analytical coherence threshold using computer simulation. Results were obtained from computer simulation that show good agreement with the theoretical estimate of the analytical coherence threshold:

$$
\gamma_{n n}^{2}=1-(1-P)^{1 /\left(n_{d}-1\right)}
$$

where we use herein $P=0.95$ and instead of the number of

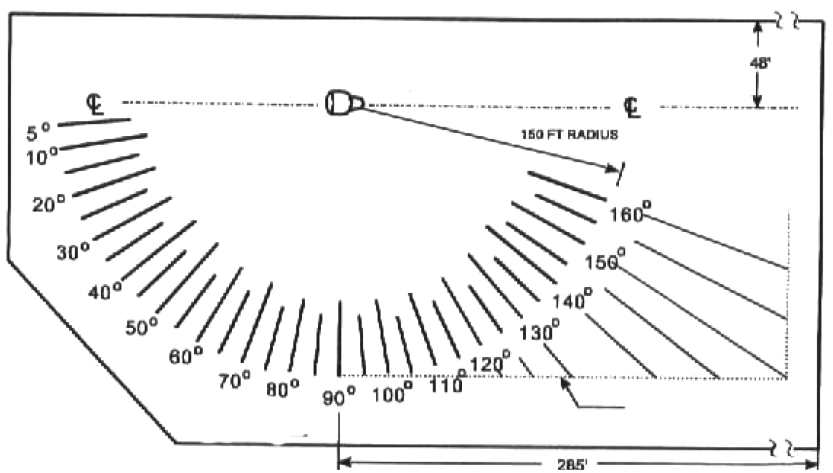

Fig. 1 Acoustic arena and microphone array at Pratt \& Whitney test stand C11, West Palm Beach, Florida, for EVNRC phase-2 tests. independent segments/blocks $n_{d}$, we take $n_{d}=n_{o}$, where $n_{o}$ is the number of overlapped segments/blocks. The value of $n_{o}$ used herein is $n_{o}=460$. Consequently, from Eq. (3), we have $\gamma_{n n}^{2}=0.0065$. The coherence threshold $\gamma_{n n}^{2}$ is discussed by Carter [38,39], Halliday et al. [40], and Brillinger [41]. The coherence threshold $\gamma_{n n}^{2}$ has a value that is greater than $95 \%$ of the values of the coherence of two independent time series calculated using $n_{d}$ disjoint data segments/blocks. The coherence threshold used herein is calculated using the number of overlapped segments, $n_{o}$. As part of the set of figures for each case used to show the results, the coherence and the threshold coherence $\gamma_{n n}^{2}$ will also be shown. The data used herein had coherence values above the threshold coherence $\gamma_{n n}^{2}$.

\section{B. Cross-Spectra Phase-Angle Sampling Errors}

In Bendat and Piersol [] ] and Piersol [42], the random error in the phase estimates due to statistical sampling is given in terms of the standard deviation of the estimated phase angle $\hat{\theta}_{12}$ by

$$
\sigma\left[\hat{\theta}_{12}(f)\right] \approx \sin ^{-1}\left\{\frac{\left[1-\gamma_{12}^{2}(f)\right]^{1 / 2}}{\left|\gamma_{12}\right| \sqrt{2 n_{o}}}\right\}
$$

where $\sigma\left[\hat{\theta}_{12}(f)\right]$ is measured in radians, and as used herein, $n_{o}$ is selected to be the number of overlapped segments or blocks used in the spectral calculations. For the special case in which the term in braces is small, Eq. (4) becomes

$$
\sigma\left[\hat{\theta}_{12}(f)\right] \approx \frac{\left[1-\gamma_{12}^{2}(f)\right]^{1 / 2}}{\left|\gamma_{12}\right| \sqrt{2 n_{o}}}
$$

where for the unknown coherence $\gamma_{12}^{2}(f)$, the estimated coherence $\hat{\gamma}_{\theta_{1} \theta_{2}}^{2}(f)$ from Eq. (2) is used. A plot of the standard deviation of the phase angle in degrees versus coherence is shown in Fig. 2.

When the coherence is greater than 0.15 , Fig. 3 shows that the standard deviation should be less than $5 \mathrm{deg}$. Straight-line fits were made to the phase-vs-frequency data. The standard deviation of the error was greater than one would calculate from the coherence (i.e., around $10 \mathrm{deg}$ when one calculated $5 \mathrm{deg}$ ). Consequently, the phaseangle measurements might be showing propagation effects due to wind shear or temperature gradients or a change in source location with frequency. In addition, the error-vs-frequency plots seemed to be in phase for each pair. As a consequence, instead of a using a fixed source location in the model, the $y$ coordinate of a source is fixed at zero and the $x$ coordinate is free to vary.

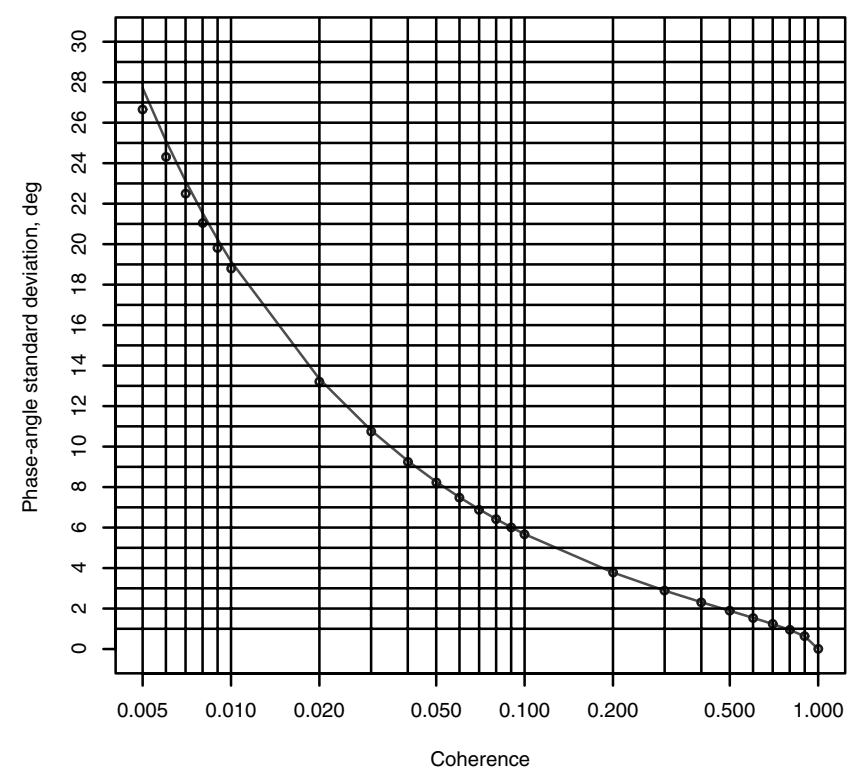

Fig. 2 Standard deviation of phase angle of $G_{34}$ based on $\gamma_{34}^{2}$ and $n_{o}$. 


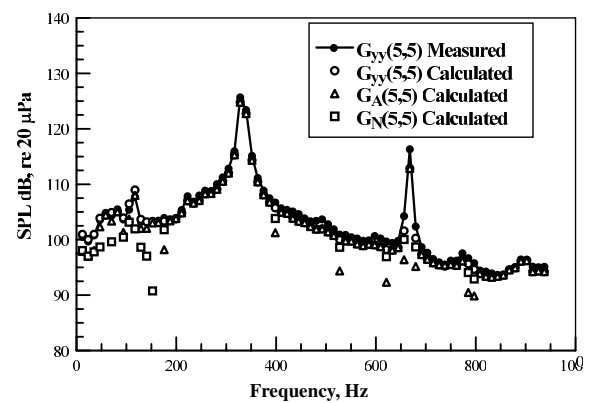

a)

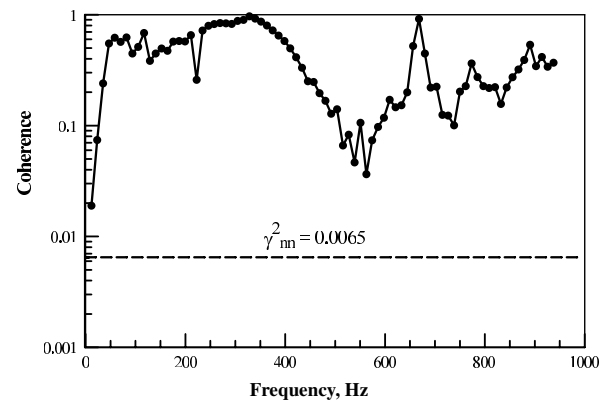

d)

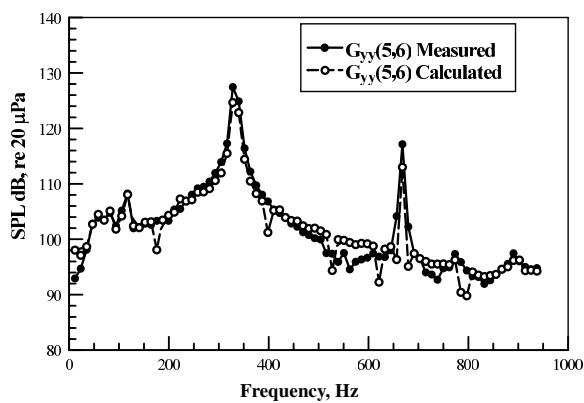

b)

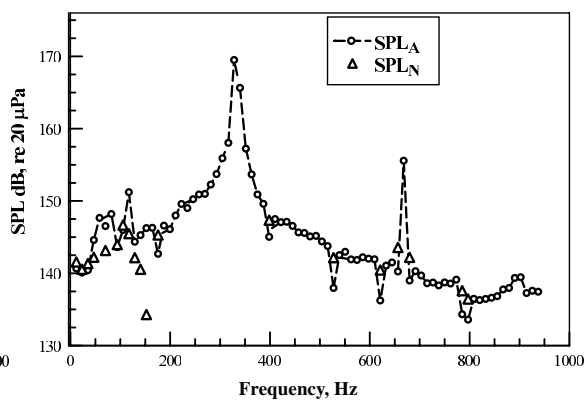

e)

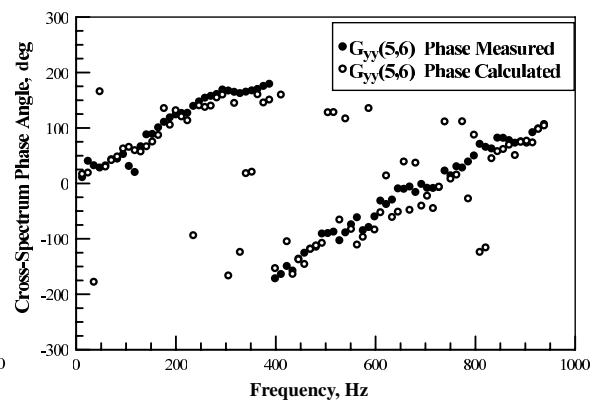

c)

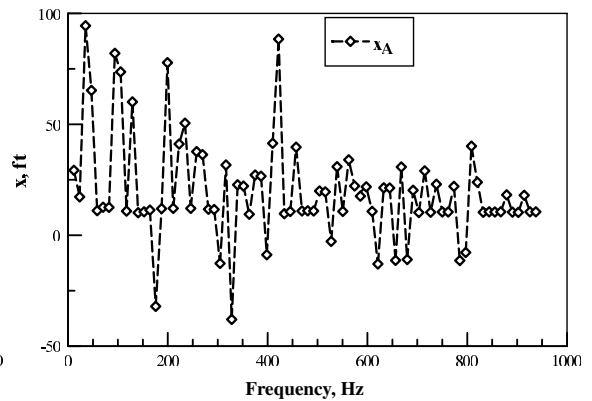

f)

Fig. 3 Three-parameter models at $150 \mathrm{ft}$ with $N 1_{\text {corr }}=582 \mathrm{rpm}$ : a) autospectrum 120-deg microphone, b) cross-spectrum magnitude between 120 - and 130-deg microphones, c) cross-spectrum phase angle between 120- and 130-deg microphones, d) coherence between 120- and 130-deg microphones, e) $\mathrm{SPL}_{A}$ and $\mathrm{SPL}_{N}$ parameters, and f) $x_{A}$ location parameter.

\section{Point-Source Models}

The method discussed was tried out over a range of operating conditions. For each case, all four models were used. Four typical cases presented herein are shown in Figs. 3-6 for $N 1_{\text {corr }}$ values of $582,1622,1900$, and $2743 \mathrm{rpm}$. Only one acoustic model result is shown for each case. The selected case uses the fewest number of parameters to achieve a good result. For each case, all four autospectra and six cross spectra are calculated. However, only the following items are shown herein:
1) The measured and calculated autospectrum is shown at $120 \mathrm{deg}$.

2) The measured and calculated cross-spectrum magnitude is shown between the 120- and 130-deg microphones.

3) The measured and calculated cross-spectrum phase angle is shown between the 120- and 130-deg microphones.

4) The measured coherence and analytic threshold coherence is shown between the 120- and 130-deg microphones.

5) The sound-pressure amplitude curve-fit parameters are shown.

6) The correlated source locations are shown.

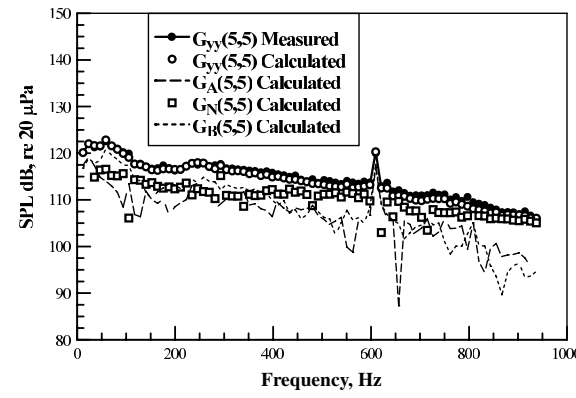

a)

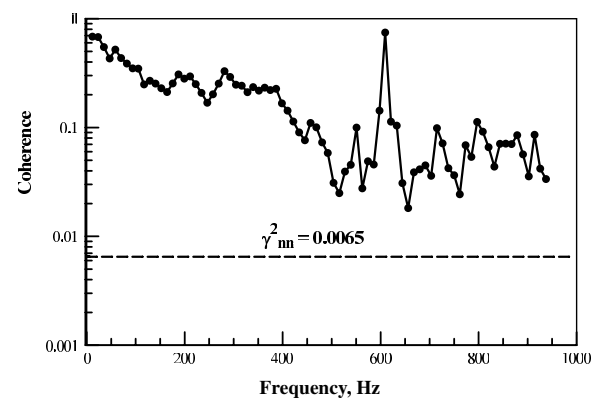

d)

Fig. 4 Five-parameter models at $150 \mathrm{ft}$ with $N 1_{\text {corr }}=1622 \mathrm{rpm}$ : a) autospectrum 120-deg microphone, b) cross-spectrum magnitude between 120 - and 130-deg microphones, c) cross-spectrum phase angle between 120- and 130-deg microphones, d) coherence between 120- and 130-deg microphones, e) $\mathrm{SPL}_{A}$ and $\mathrm{SPL}_{N}$ parameters, and f) $x_{A}$ location parameter.

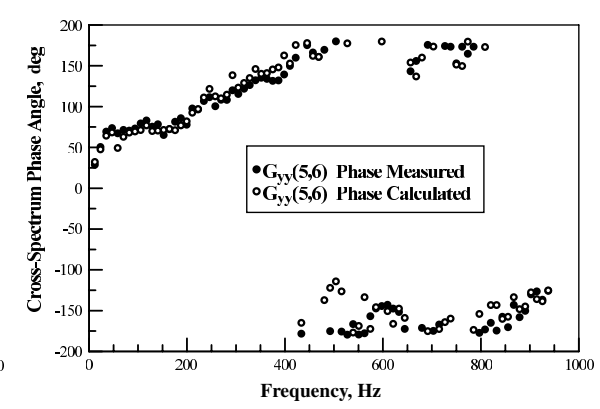

c)
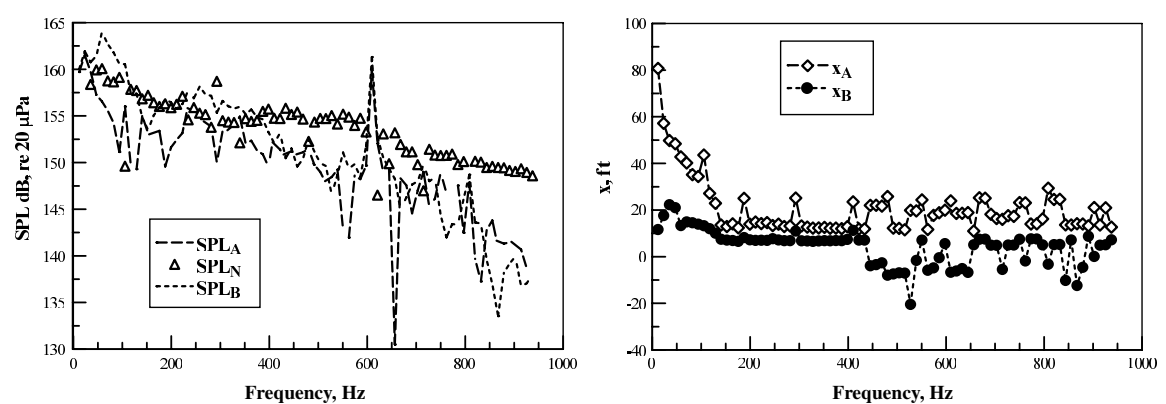

f) 


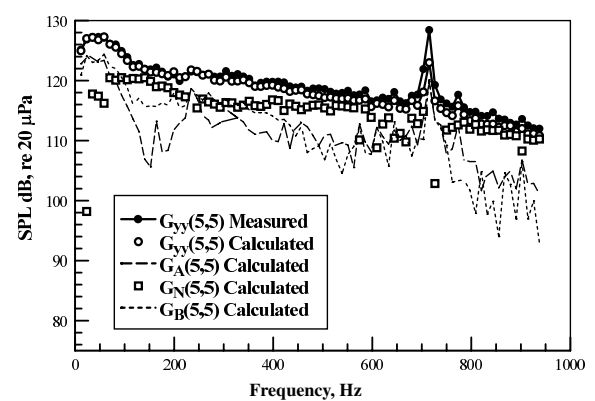

a)

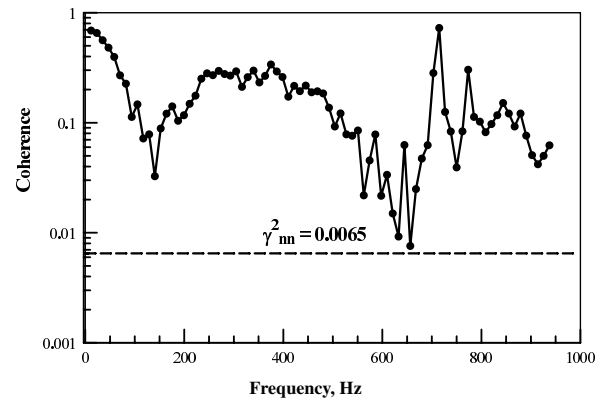

d)

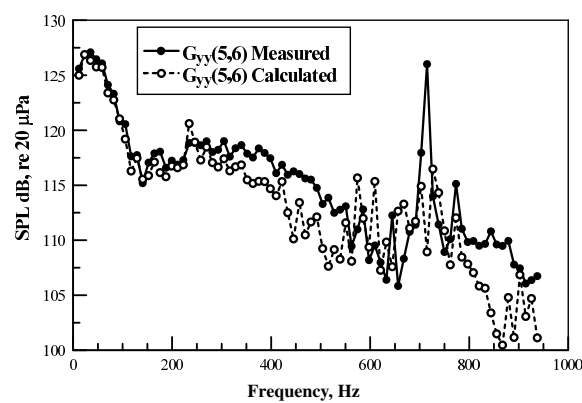

b)

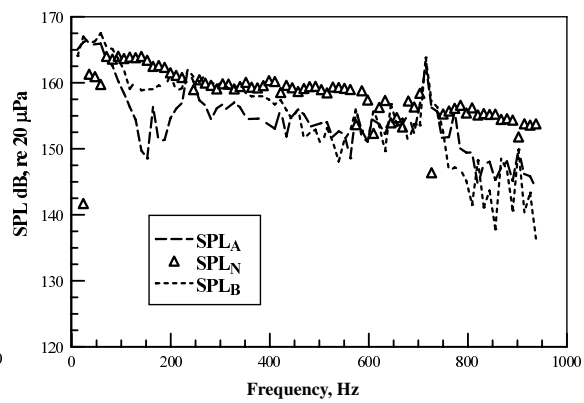

e)

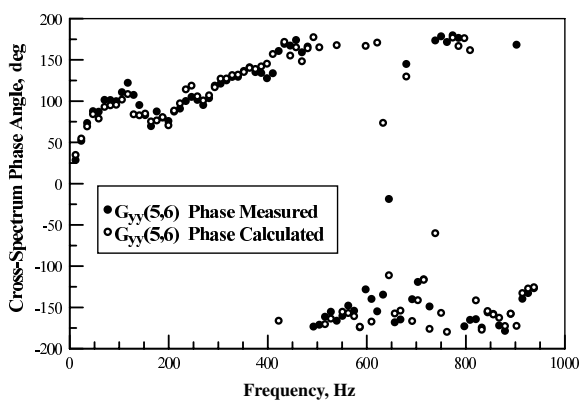

c)

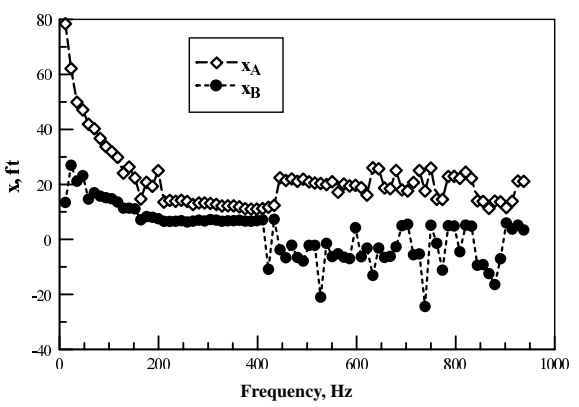

f)

Fig. 5 Five-parameter models at $150 \mathrm{ft}$ with $N 1_{\text {corr }}=1900 \mathrm{rpm}$ : a) autospectrum 120-deg microphone, b) cross-spectrum magnitude between 120 - and 130-deg microphones, c) cross-spectrum phase angle between 120- and 130-deg microphones, d) coherence between 120- and 130-deg microphones, e) $\mathrm{SPL}_{A}$ and $\mathrm{SPL} \mathrm{L}_{N}$ parameters, and f) $x_{A}$ location parameter.

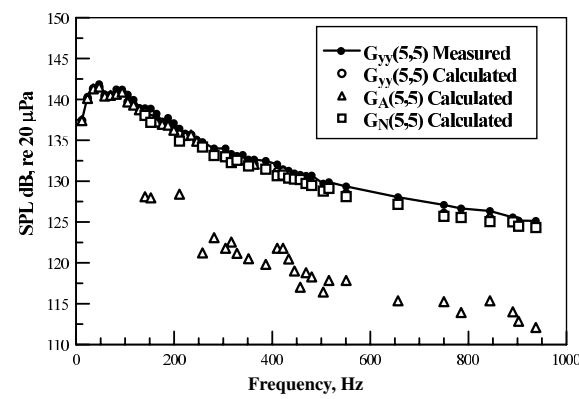

a)

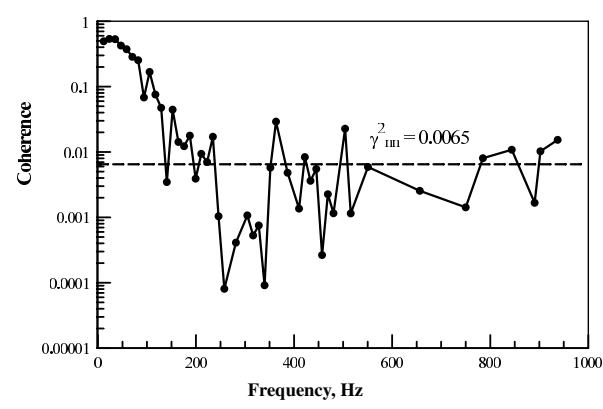

d)

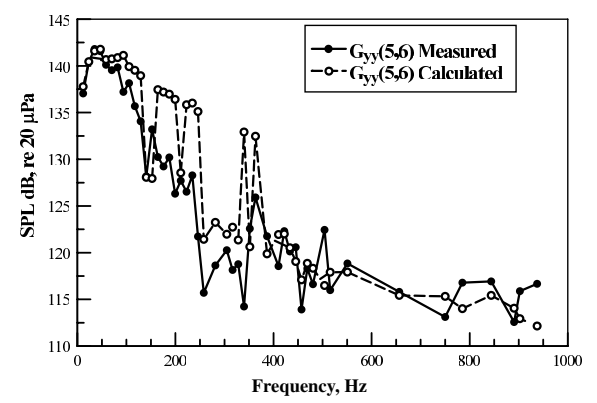

b)

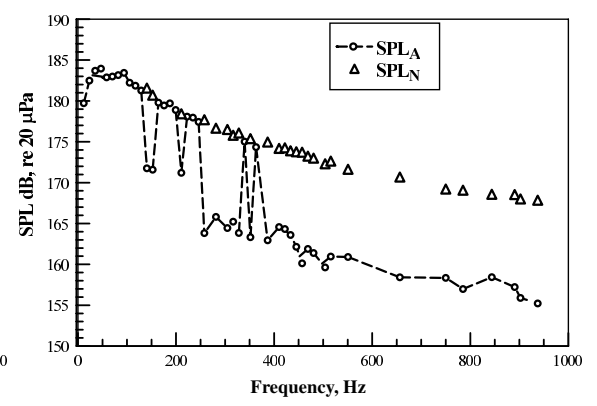

e)

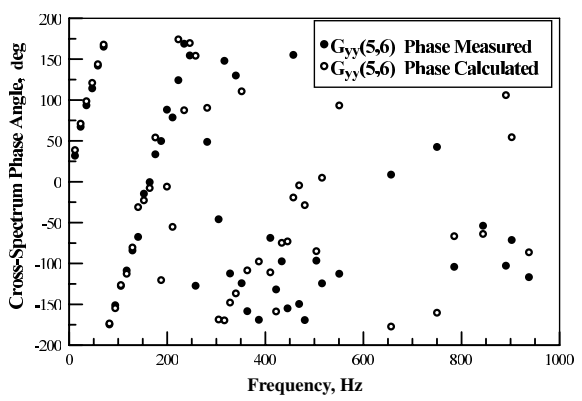

c)

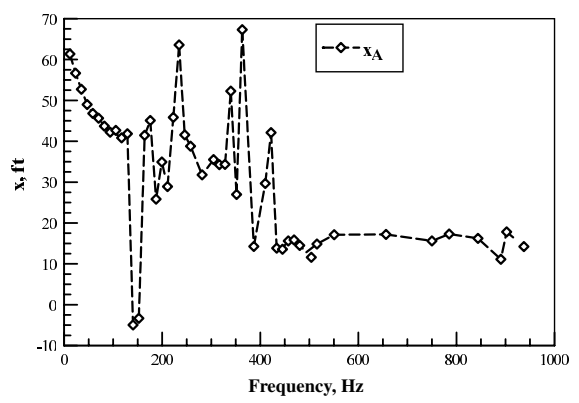

f)

Fig. 6 Three-parameter models at $150 \mathrm{ft}$ with $N 1_{\text {corr }}=2743 \mathrm{rpm}$ : a) autospectrum 120-deg microphone, b) cross-spectrum magnitude between 120 and 130-deg microphones, c) cross-spectrum phase angle between 120- and 130-deg microphones, d) coherence between 120- and 130-deg microphones, e) $\mathrm{SPL}_{A}$ and $\mathrm{SPL} \mathrm{L}_{N}$ parameters, and f) $x_{A}$ location parameter.

In the modeling scheme used herein, for each case, the jet noise was initially assumed to be uncorrelated between the microphones at all frequencies. The results show that this assumption may be true at frequencies greater than $164 \mathrm{~Hz}$. Furthermore, it is assumed the basic radiation pattern of the correlated noise as specified by the crossspectrum phase angle could be represented by a single coherent point source at some $x$ location. In all cases, a better result is obtained by using an acoustic model that assumes that an uncorrelated noise source is present and letting the computer solution procedure determine if its value is significant. In some cases, a second coherent point source gives a slightly better result. Only the coherent sources create the cross spectra. The autospectra are due to the coherent and incoherent sources.

The general formulation is given in Appendix A. The general models are shown in Appendices $\underline{B}, \underline{C}, \underline{D}$, and $\underline{E}$

\section{1. $N 1_{\text {corr }}=582 \mathrm{rpm}$}

At the engine-idle $N 1_{\text {corr }}=582$-rpm operating condition, the core noise is dominated by a low-frequency combustion instability tone at $327 \mathrm{~Hz}$, as shown in Fig. 3a, in which the 120-deg autospectrum is shown. Results are shown for the three-parameter doublet-source 
model $\left(A, N\right.$, and $\left.x_{A}\right)$. The noise radiation pattern can be represented by a single point source, as shown in Figs. $3 \mathrm{~b}$ and $3 \mathrm{c}$, in which the cross spectrum between the 120- and 130-deg microphones are shown. This cross spectrum is shown because the coherence calculated using the 120- and 130-deg microphones is above 0.04 , as shown in Fig. 3d, and the phase-angle error is less than 9 deg (using Fig. 2). The results shown are for the three-parameter couplet-source model $\left(A, N\right.$, and $\left.x_{A}\right)$ described in Appendix C. As shown in Fig. 3a, at a few low-frequency points, the computer solution included $\bar{a}$ uncorrelated noise source. The coherence shown in Fig. $3 \mathrm{~d}$ is above the coherence threshold $\gamma_{n n}^{2}=0.0065$, shown as a dashed line in Fig. 3d. However, the coherence shows a lot of variation above $400 \overline{\mathrm{Hz}}$, which signifies a greater statistical deviation in the phase angle and more uncertainty in the location solutions. The values of $\mathrm{SPL}_{A}$ and $\mathrm{SPL}_{N}$ are shown in Fig. 3e. At most points, the uncorrelated noise level was too low to plot, as shown in Fig. $3 \mathrm{e}$. As shown in Fig. 3f, to match the measured phase angle, the computer tends to find solutions in which $x_{A}=10 \mathrm{ft}$ at low frequencies and $20<x_{A}<40$ at higher frequencies.

\section{2. $N 1_{\text {corr }}=1622 \mathrm{rpm}$}

At the $N 1_{\text {corr }}=1622$-rpm operating condition, results are shown for the five-parameter triplet-source model $\left(A, B, N, x_{A}\right.$, and $\left.x_{B}\right)$ described in Appendix E. As shown in Fig. 4a, this model does a good job of representing the 120-deg microphone measured autospectrum. The noise radiation pattern is well-represented by two point sources, as shown in Figs. $4 \mathrm{~b}$ and $4 \mathrm{c}$, in which the magnitude and phase of the cross spectrum between the 120- and 130-deg microphones are shown. Below $400 \mathrm{~Hz}$, this model does a fine job. The coherence between the 120- and 130-deg microphones shown in Fig. $4 \mathrm{~d}$ is above the coherence threshold $\gamma_{n n}^{2}=0.0065$. Below $400 \mathrm{~Hz}$, the coherence is greater than 0.10 . Consequently, the cross-spectrum phase angles look good below $400 \mathrm{~Hz}$.

The source solution SPL values are shown Fig. 4e, and the source solution location values are shown in Fig. 4f. The variation of the source- $A$ location solution below $164 \mathrm{~Hz}$ corresponds to the change in thelocation of a low-frequency noise source from near the end of the potential core of the jet toward the nozzle as higher-frequency solutions are found. Consequently, we associate $\mathrm{SPL}_{A}$ with the jet source in the frequency range below $164 \mathrm{~Hz}$.

The variation of the source amplitude of the solution for source $B$ shown in Fig. 4e and the variation of the far-field $\mathrm{SPL}_{B}$ autospectra shown in Fig. 4 a have the same frequency dependence that the combustion modes have, in that they seem to be peaking in the 0 - to $200-\mathrm{Hz}$ band and the $200-$ to $400-\mathrm{Hz}$ band. This characteristic behavior was reported in a study of PW4098 annular combustor modes by Miles [3] . This suggests that the correlated noise source $B$ is associated with the combustor source in the 0 - to $400-\mathrm{Hz}$ frequency range. The source locations of the two point sources shown in Fig. $4 \mathrm{f}$ have an interesting symmetry that will be discussed in the next section.

\section{3. $N 1_{\text {corr }}=1900 \mathrm{rpm}$}

Again, at the $N 1_{\text {corr }}=1900$-rpm operating condition, results are shown for the five-parameter triplet-source model $\left(A, B, N, x_{A}\right.$, and $x_{B}$ ) described in Appendix E. As shown in Fig. 5a, this model does a good job of representing the 120-deg microphone measured autospectrum.

The noise radiation pattern is well-represented by two point sources, as shown in Figs. $\underline{5 b}$ and $\underline{5 c}$, in which the magnitude and phase of the cross spectrum between the 120- and 130-deg microphones are shown. The coherence shown in Fig. $5 \mathrm{~d}$ is above the coherence threshold $\gamma_{n n}^{2}=0.0065$ and greater than 0.04 at most frequencies less than $500 \mathrm{~Hz}$. Consequently, the cross-spectrum phase angles look good below $500 \mathrm{~Hz}$.

The source-solution SPL values are shown Fig. 5e and the sourcesolution location values are shown in Fig. 5f. Again, the variation of the source- $A$ location solution below $16 \overline{4} \mathrm{~Hz}$ corresponds to the change in location of a low-frequency noise source from near the end of the potential core of the jet toward the nozzle as higher-frequency solutions are found. Consequently, we again associate $\mathrm{SPL}_{A}$ with the jet source in the frequency range below $164 \mathrm{~Hz}$.

Again, the variation of the source amplitude of the solution for source $B$ shown in Fig. $\underline{5 \mathrm{e}}$ and the variation of the far-field $\mathrm{SPL}_{B}$ autospectra shown in Fig. 5 a have the same frequency dependence that the combustion modes have, in that they seem to be peaking in the 0 - to $200-\mathrm{Hz}$ band and the 200- to $400-\mathrm{Hz}$ band. This characteristic behavior was reported in a study of PW4098 annular combustor modes by Miles [3]. This suggests that the correlated noise source $B$ is associated with the combustor source in the 0 - to 400-Hz frequency range.

The source locations of the two point sources shown in Fig. $\underline{4 f}$ have an interesting symmetry that will be discussed in the next section.

\section{4. $\quad N 1_{\text {corr }}=2743 \mathrm{rpm}$}

The highest power setting examined herein is the $N 1_{\text {corr }}=$ 2743 -rpm operating condition. The autospectrum at $120 \mathrm{deg}$ is shown in Fig. 6a. Below $100 \mathrm{~Hz}$, the uncorrelated noise source is not needed to represent the measurements. The noise radiation pattern is well-represented again below $100 \mathrm{~Hz}$ by a single point source, as shown in Figs. $\underline{6 b}$ and $\underline{6 c}$. In these figures, the magnitude and phase of the cross spectrum between the 120- and 130-deg microphones are shown. The results shown are for the three-parameter couplet-source model $\left(A, N\right.$, and $\left.x_{A}\right)$ described in Appendix $\underline{C}$. As shown in Fig. 6a, at frequencies above $100 \mathrm{~Hz}$, the computer solution included an uncorrelated noise source. The coherence shown in Fig. 6d is above the coherence threshold $\gamma_{n n}^{2}=0.0065$ below $100 \mathrm{~Hz}$. Below $100 \mathrm{~Hz}$, the coherence is above 0.05 . Consequently, the cross-spectrum phase angles look good below $100 \mathrm{~Hz}$.

Above $200 \mathrm{~Hz}$, the coherence is that of two random signals, except at select points. Consequently, the phase angle shown in Fig. $\underline{6 c}$ preceding $200 \mathrm{~Hz}$ is very random. The autospectrum solution shown in Fig. 6a looks good because the solution is based on mostly the incoherent level SPL $\mathrm{SP}_{N}$. Below $100 \mathrm{~Hz}$, the uncorrelated noise level was too low to plot, as shown in Fig. 6e. As shown in Fig. 6f, below $100 \mathrm{~Hz}$, the decrease in the value of the location of source $A$ with frequency shows that source $A$ can again be interpreted as being due to low-frequency sound radiation from the jet, as was the case with the $x_{A}$ results shown in Figs. $4 \mathrm{f}$ and $5 \mathrm{f}$. Again, this corresponds to the change in location of a low-frequency noise source from near the end of the potential core of the jet toward the nozzle as higher-frequency solutions are found.

\section{Source Location}

To provide further information on the source-location parameter, statistical distribution studies were conducted. In addition, for the five-parameter cases, scatter plots of $x_{A}$ and $x_{B}$ indexed by the frequency sequence number were made. These results are discussed in this section.

\section{A. $\quad N 1_{\text {corr }}=582 \mathrm{rpm}$}

Histogram and nonparametric kernel-density estimates [43] of the distribution of location parameter $x_{A}$ are shown in Fig. 7. This plot also shows that the computer tends to find solutions in which $x_{A}=10 \mathrm{ft}$ at low frequencies and $20<x_{A}<40$ at higher frequencies. The nozzle in this geometry appears to be near $x_{A}=10 \mathrm{ft}$.

The greater phase-angle standard deviation in the frequency range above $400 \mathrm{~Hz}$ has increased the uncertainty of the location parameter for source $A, x_{A}$.

\section{B. $N 1_{\text {corr }}=1622 \mathrm{rpm}$}

A scatter plot of $x_{A}$ and $x_{B}$ identifying frequency with the index number is shown in Fig. 8a. The movement of source location $x_{A}$ from near the end of the jet potential core toward the nozzle as the frequency is increased is clearly shown. At low frequencies, the two major sources are the core noise and the jet noise. Above $400 \mathrm{~Hz}$, the coherence becomes more irregular and the cross-spectrum phase 


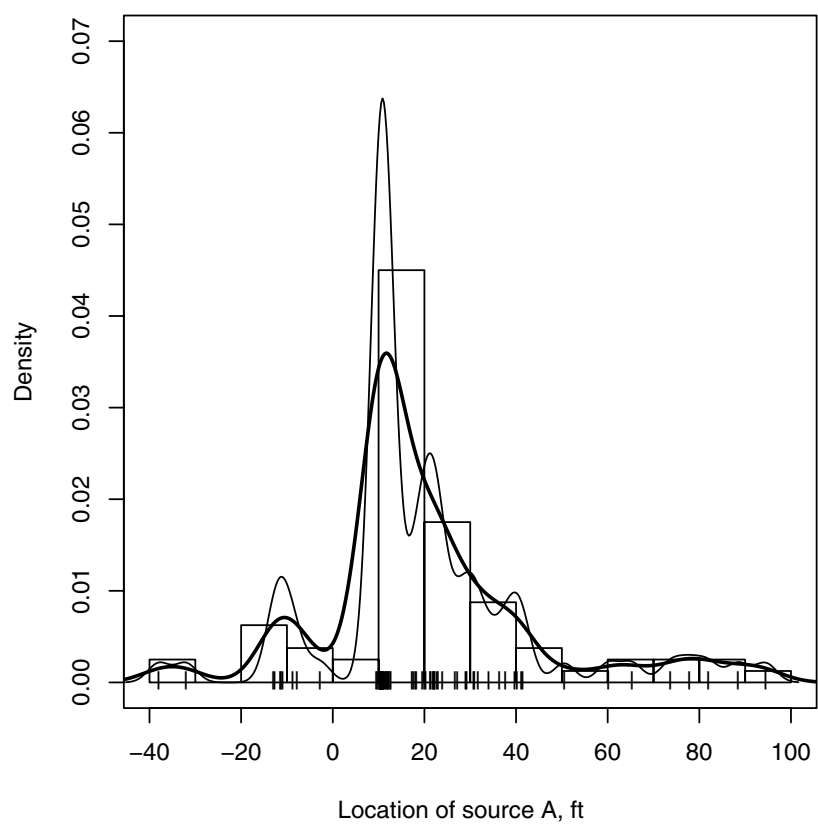

Fig. 7 Histogram and nonparametric kernel-density estimates of the distribution of location parameter $x_{A}$, using the default bandwidth (heavier line) and half the default bandwidth (lighter line) based on a three-parameter model; A one-dimensional scatter plot (or rug plot) is shown at the bottom of the graph; $N 1_{\text {corr }}=582 \mathrm{rpm}$. becomes more random. Consequently, the location results are more unreliable. They are shown in the scatter plot but are not very useful.

Figures $8 \mathrm{~b}$ and $8 \mathrm{c}$ show a histogram and nonparametric kerneldensity estimates of the distribution of location parameter $x_{A}$ using the default bandwidth (heavier line) and half the default bandwidth (lighter line) for 0-152 and 164-937 Hz. A one-dimensional scatter plot (or rug plot) is shown at the bottom of the graphs. For the frequency range of $0-152 \mathrm{~Hz}$ shown in Fig. $8 \mathrm{~b}$, the distribution is flat with a concentration near $40 \mathrm{ft}$. For the frequency range of 164 $937 \mathrm{~Hz}$ shown in Fig. 8c, the distribution has peaks between 11 and $15 \mathrm{ft}$.

Figures $8 \mathrm{~d}$ and $8 \mathrm{e}$ show a histogram and nonparametric kerneldensity estimates of the distribution of location parameter $x_{B}$ using the default bandwidth (heavier line) and half the default bandwidth (lighter line) for two frequency ranges: 0-152 and 164-937 Hz. A one-dimensional scatter plot (or rug plot) is shown at the bottom of the graphs. For the frequency range of $0-152 \mathrm{~Hz}$ shown in Fig. 8d, the distribution peaks between 11 and $15 \mathrm{ft}$. For the frequency range of $164-937 \mathrm{~Hz}$ shown in Fig. 8e, the distribution has one peak near $-5 \mathrm{ft}$ and another near $7 \mathrm{ft}$.

\section{C. $\quad N 1_{\text {corr }}=1900 \mathrm{rpm}$}

A scatter plot of $x_{A}$ and $x_{B}$ identifying frequency with the index number is shown in Fig. 9a. The movement of source location $x_{A}$ from near the end of the jet potential core toward the nozzle as the frequency is increased is clearly shown. At low frequencies, the two major sources are the core noise and the jet noise. Again, above $400 \mathrm{~Hz}$, the coherence becomes more irregular and cross-spectrum

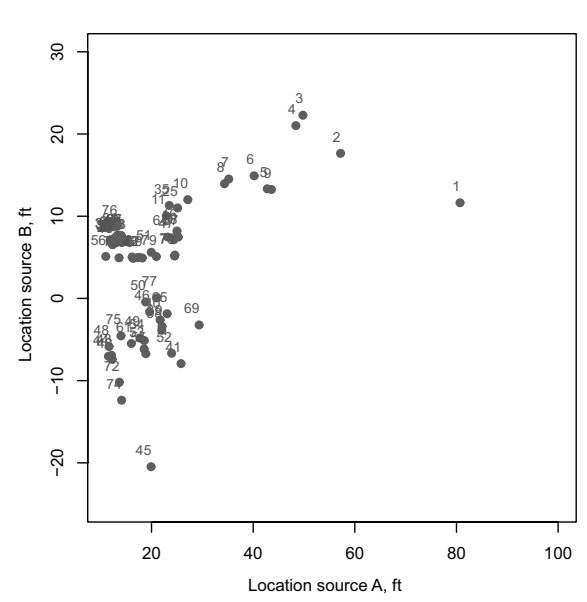

a)

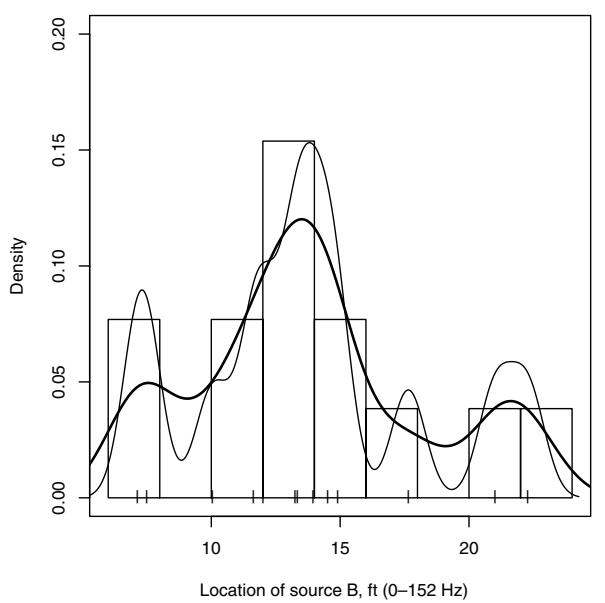

d)

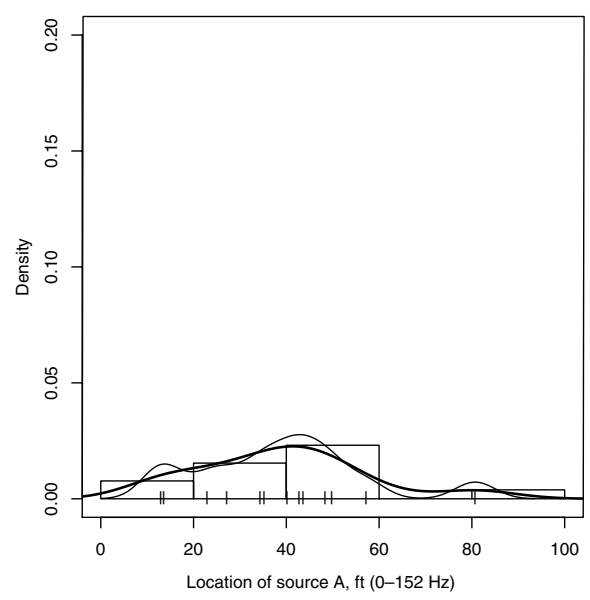

b)

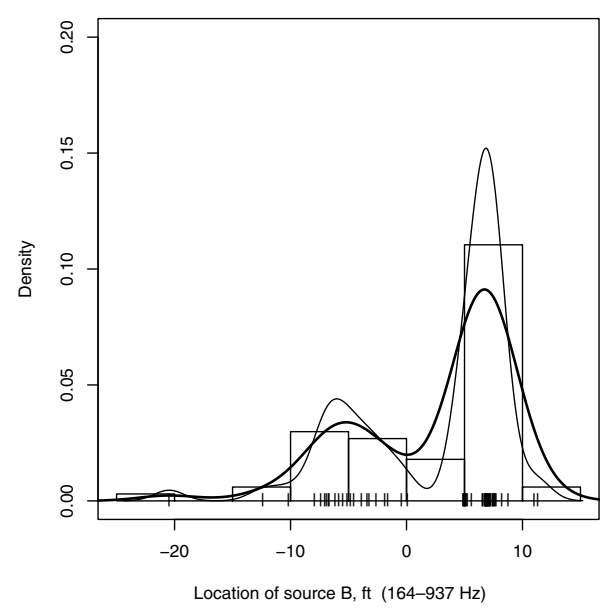

e)

Fig. 8 Five-parameter model at $150 \mathrm{ft}$ with $N 1_{\text {corr }}=1622 \mathrm{rpm}$ : a) scatter plot of $x_{A}$ by $x_{B}$ coded by frequency index for the whole region (164-937 Hz), b) histogram and nonparametric kernel-density estimates of the distribution of location parameter $x_{A},(0-152 \mathrm{~Hz})$, c) histogram and nonparametric kernel-density estimates of the distribution of location parameter $x_{A}(164-937 \mathrm{~Hz})$, d) histogram and nonparametric kernel-density estimates of the distribution of location parameter $x_{B}(0-152 \mathrm{~Hz})$, and e) histogram and nonparametric kernel-density estimates of the distribution of location parameter $x_{B}(164-937 \mathrm{~Hz})$. 


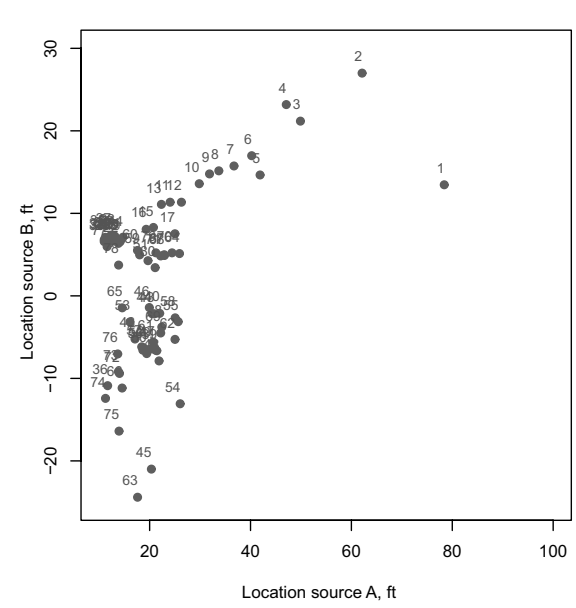

a)

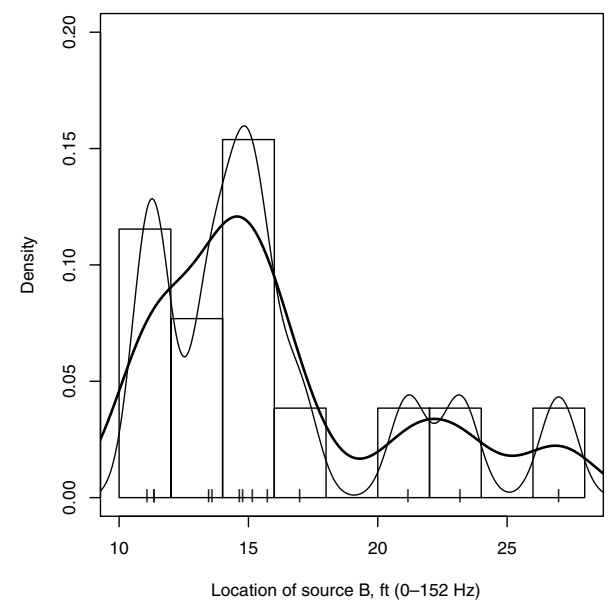

d)

b)
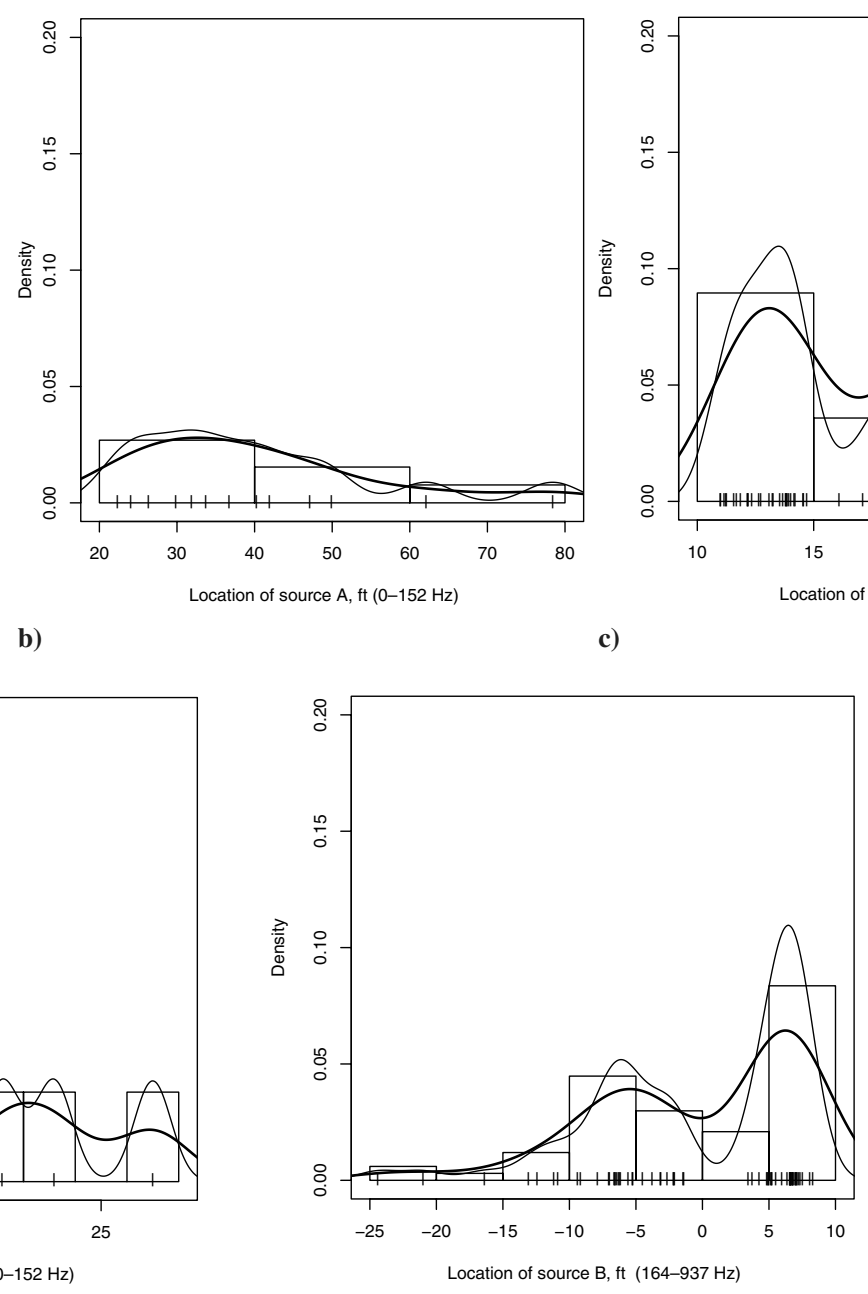

e)

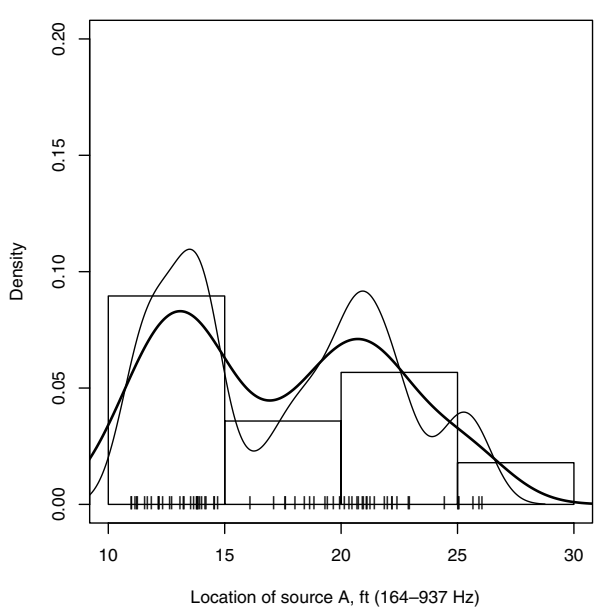

c)

Fig. 9 Five-parameter model at $N 1_{\text {corr }}=1900 \mathrm{rpm}$ : a) scatter plot of $x_{A}$ by $x_{B}$ coded by frequency index for the whole region (164-937 Hz), b) histogram and nonparametric kernel-density estimates of the distribution of location parameter $x_{A}(0-152$ Hz), c) histogram and nonparametric kernel-density estimates of the distribution of location parameter $x_{A}(164-937 \mathrm{~Hz})$, d) histogram and nonparametric kernel-density estimates of the distribution of location parameter $x_{B}(0-152 \mathrm{~Hz})$, and e) histogram and nonparametric kernel-density estimates of the distribution of location parameter $x_{B}(164-937 \mathrm{~Hz})$.

phase becomes more random. Consequently, the location results are more unreliable. They are shown in the scatter plot but are not very useful.

Figures $9 \mathrm{~b}$ and $9 \mathrm{c}$ show a histogram and nonparametric kerneldensity estimates of the distribution of location parameter $x_{A}$ using the default bandwidth (heavier line) and half the default bandwidth (lighter line) for two frequency ranges: 0-152 and 164-937 Hz. A one-dimensional scatter plot (or rug plot) is shown at the bottom of the graphs. For the frequency range of $0-152 \mathrm{~Hz}$ shown in Fig. 9b, the distribution is flat with a concentration near $30 \mathrm{ft}$. For the frequency range of 164-937 Hz shown in Fig. 9c, the distribution has one peak near $20 \mathrm{ft}$ and another near $13 \mathrm{ft}$.

Figures $9 \mathrm{~d}$ and $9 \mathrm{e}$ show a histogram and nonparametric kerneldensity estimates of the distribution of location parameter $x_{B}$ using the default bandwidth (heavier line) and half the default bandwidth (lighter line) for two frequency ranges: 0-152 and 164-937 Hz. A one-dimensional scatter plot (or rug plot) is shown at the bottom of the graphs. For the frequency range of $0-152 \mathrm{~Hz}$ shown in Fig. 9d, the distribution peaks near $15 \mathrm{ft}$. For the frequency range of $1 \overline{64}$ $937 \mathrm{~Hz}$ shown in Fig. 9e, the distribution has one peak near $-7 \mathrm{ft}$ and another near $7 \mathrm{ft}$.

\section{D. $N 1_{\text {corr }}=2743 \mathrm{rpm}$}

Histogram and nonparametric kernel-density estimates of the distribution of location parameter $x_{A}$ are shown in Fig. 10. This plot also shows that the computer tends to find solutions in which
$x_{A}=10 \mathrm{ft}$ at low frequencies and $20<x_{A}<40$ at higher frequencies. The nozzle in this geometry appears to be near $x_{A}=10 \mathrm{ft}$.

The phase-angle randomness in the frequency range above $100 \mathrm{~Hz}$ has increased the uncertainty of the location parameter for source $A$, $x_{A}$.

\section{Discussion}

The solution procedure provides location and sound-pressurelevel values. The results just presented indicate that one can use the model solutions to separate the jet noise and the combustion noise source provided the coherence between the microphones is high enough to reduce the standard deviation of the phase angle.

For the $N 1_{\text {corr }}$ cases of 1622,1900 , and $2743 \mathrm{rpm}$, the procedure tracks the jet-mixing-noise source location as a function of frequency. These results are in good agreement with those obtained using linear phased microphone arrays to study the source distribution of a Rolls-Royce/SNECMA Olympus engine, as presented by Flynn and Kinns [27] (Figs. 6 and 7) and by Billingsley and Kinns [28] (Fig. 13).

In addition, for the $N 1_{\text {corr }}$ cases of 1622 and $1900 \mathrm{rpm}$, the procedure identifies the core-noise source at the nozzle exit. These results are in good agreement with those using the polar correlation technique of Fisher et al. [29] (Figs. 15 and 16), in which a source distribution of a Viper $\overline{601}$ is shown and in which a source 


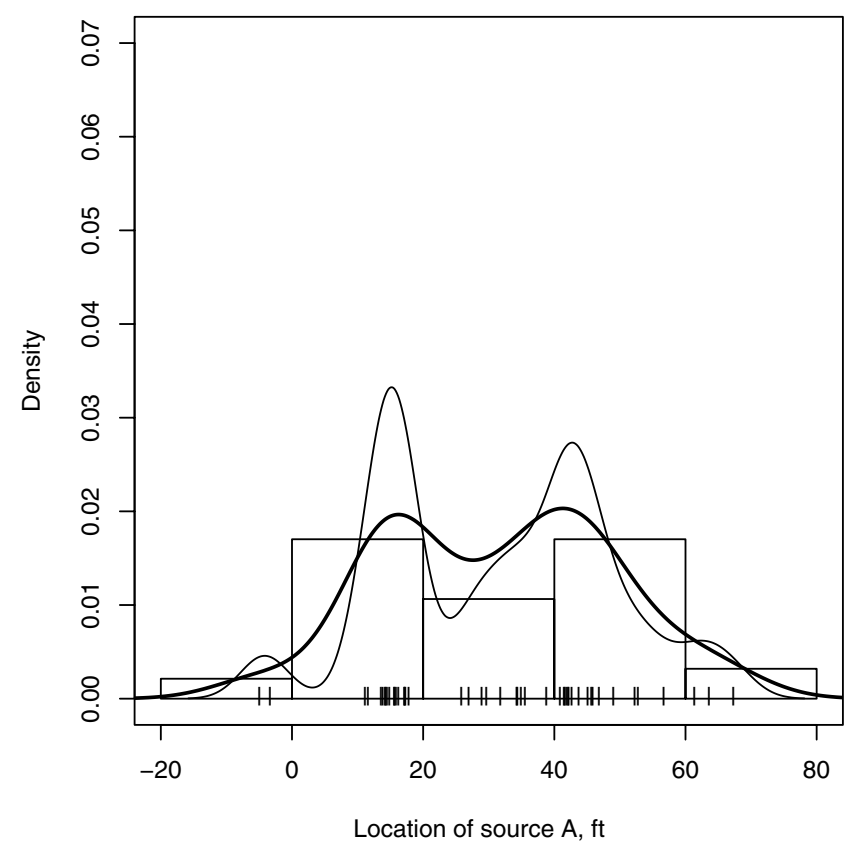

Fig. 10 Histogram and nonparametric kernel-density estimates of the distribution of location parameter $x_{A}$, using the default bandwidth (heavier line) and half the default bandwidth (lighter line) based on a three-parameter model; a one-dimensional scatter plot (or rug plot) is shown at the bottom of the graph; $N 1_{\text {corr }}=2743 \mathrm{rpm}$.

distribution of a RP211 is shown. In addition, they are similar to the results shown by Siller et al. [22] (Fig. 17), who used a phasedmicrophone array to study BR700 engine noise.

These results show how important it is to start with no preconceived idea of what acoustic-source mode best represents the measured turbojet core noise. One should use a model that best fits the data and not one's idea of the model.

The results show that the uncorrelated noise source is important. Furthermore, the results show that the coherent core noise is greater than the coherent jet noise at frequencies less than $400 \mathrm{~Hz}$ at these angles.

The use of four microphones separated by $10 \mathrm{deg}$ in the method makes the procedure applicable to many sets of data taken in the normal study of engine noise at engine test stands. This makes this source-separation procedure easier to apply than other microphonearray methods that require special close microphone spacing and many more microphones $[22,28,29,27]$ or the binaural sourcelocation procedure of Kinns [31], which requires close microphone spacing. In addition, the source-separation method might be applicable to the core-noise-source modeling portion of the Aircraft Noise Prediction Program (ANOPP) [44].

\section{Conclusions}

A method to separate correlated signals buried in turbofan engine core noise was presented. The method is based on finding acoustic model coefficients that enable a system of equations based on one or two correlated monopole noise sources and an uncorrelated monopole noise source to reproduce experimental data.

At the lower engine-power settings, the nozzle exit is the strongest noise source. At the higher engine-power settings, the jet noise is observable, but the core noise is not observable because the coherence between the microphone becomes too low and the phaseangle measurements become meaningless. Above $164 \mathrm{~Hz}$, at the higher engine-power setting, one has only an uncorrelated noise source.

The effectiveness and the reliability of the method have been tested using Pratt \& Whitney PW4098 far-field acoustic measurements. The agreement achieved between the experimental data and the acoustic model used confirm the effectiveness of the procedure.

\section{Appendix A: One-Sided Spectrum Functions for Acoustic Signals from a Point Source}

We shall assume a compact source region and assume that the source produces a wave that spreads spherically outward with no preferred direction. The wave equation in this case, as discussed by Morse and Ingard [45] (page 309), is

$$
\frac{1}{r^{2}} \frac{\partial}{\partial r}\left(r^{2} \frac{\partial p}{\partial r}\right)=\frac{1}{c_{o}^{2}} \frac{\partial^{2} p}{\partial t^{2}}
$$

We shall assume a simple source solution to the wave equation:

$$
p(t)=\frac{A}{R} \exp (-i k R) \sin \left(2 \pi f_{0} t\right)
$$

where

$$
\begin{gathered}
k=\frac{2 \pi f_{0}}{c_{o}} \quad c_{o}^{2}=\frac{\gamma \Re T K}{M W} \mathrm{~m} / \mathrm{s} \quad \Re=8314 \mathrm{~J} / \mathrm{k} \mathrm{mol} \mathrm{K} \\
M W=28.97 \mathrm{~kg} / \mathrm{kmol} \quad T F=80
\end{gathered}
$$

To get the autospectrum and cross spectrum of a point source, we use the following relationships from correlation and spectral analysis texts by Bendat and Piersol [4-6].

The autocorrelation function of $x$ is

$$
R_{x x}(\tau)=E\left[x_{k}(t) x_{k}(t+\tau)\right]
$$

The autocorrelation function for a sum of two processes $y(t)=$ $x_{1}(t)+x_{2}(t)$ is

$$
\begin{gathered}
R_{y y}(\tau)=E\left\{\left[x_{1}(t)+x_{2}(t)\right]\left[x_{1}(t+\tau)+x_{2}(t+\tau)\right]\right\} \\
\quad=R_{x_{1} x_{1}}(\tau)+R_{x_{1} x_{2}}(\tau)+R_{x_{2} x_{1}}(\tau)+R_{x_{2} x_{2}}(\tau)
\end{gathered}
$$

The two-sided auto- and cross-spectral density functions are

$$
\begin{aligned}
& S_{x x}(f)=F\left[R_{x x}(\tau)\right]=\int_{-\infty}^{\infty} R_{x x}(\tau) \exp (-j 2 \pi f \tau) \mathrm{d} \tau \\
& S_{x y}(f)=F\left[R_{x y}(\tau)\right]=\int_{-\infty}^{\infty} R_{x y}(\tau) \exp (-j 2 \pi f \tau) \mathrm{d} \tau
\end{aligned}
$$

and of course we have the inverse relationships

$$
\begin{aligned}
& R_{x x}(\tau)=F^{-1}\left[S_{x x}(f)\right]=\int_{-\infty}^{\infty} S_{x x}(f) \exp (+j 2 \pi f \tau) \mathrm{d} f \\
& R_{x y}(\tau)=F^{-1}\left[S_{x y}(f)\right]=\int_{-\infty}^{\infty} S_{x y}(f) \exp (+j 2 \pi f \tau) \mathrm{d} f
\end{aligned}
$$

The two-sided autospectrum function for a sum of two processes $y(t)=x_{1}(t)+x_{2}(t)$ is

$$
\begin{aligned}
& S_{y y}(f)=F\left[E\left\{\left[x_{1}(t)+x_{2}(t)\right]\left[x_{1}(t+\tau)+x_{2}(t+\tau)\right]\right\}\right] \\
& \quad=F\left[R_{x_{1} x_{1}}(\tau)+R_{x_{1} x_{2}}(\tau)+R_{x_{2} x_{1}}(\tau)+R_{x_{2} x_{2}}(\tau)\right] \\
& \quad=S_{x_{1} x_{1}}(f)+S_{x_{1} x_{2}}(f)+S_{x_{2} x_{1}}(f)+S_{x_{2} x_{2}}(f)
\end{aligned}
$$

The autocorrelation function is the Fourier transform of the correlation of $x(t)$ with itself and is related to the Fourier transform of $x(t)$ by

$$
S_{x x}(f)=F\left[R_{x x}(\tau)\right]=\int_{-\infty}^{\infty} R_{x x}(\tau) \exp (-j 2 \pi f \tau) \mathrm{d} \tau=X(f) X^{*}(f)
$$


The cross-correlation function is the Fourier transform of the correlation of $x(t)$ and $y(t)$ and is related to the Fourier transform of $x(t)$ and $y(t)$ by

$$
S_{x y}(f)=F\left[R_{x y}(\tau)\right]=\int_{-\infty}^{\infty} R_{x y}(\tau) \exp (-j 2 \pi f \tau) \mathrm{d} \tau=X(f) Y^{*}(f)
$$

The one-sided spectral density function is $G_{x}(f)=2 S_{x}(f)$ $0 \leq f<\infty$. is

The autocorrelation function of a sine wave $x(t)=A \cos \left(2 \pi f_{0} t\right)$

$$
R_{x x}(\tau)=\frac{A^{2}}{2} \cos \left(2 \pi f_{0} \tau\right)
$$

The power spectral density of a sine wave $x(t)=A \cos \left(2 \pi f_{0} t\right)$ is

$$
G_{x x}(f)=\frac{A^{2}}{2} \delta\left(f-f_{0}\right)
$$

The one-sided spectrum function for a sum of two processes $y(t)=$ $x_{1}(t)+x_{2}(t)$ is

$$
G_{y y}(f)=G_{x_{1} x_{1}}(f)+G_{x_{1} x_{2}}(f)+G_{x_{2} x_{1}}(f)+G_{x_{2} x_{2}}(f]
$$

Next, these relationships are applied to signals produced by acoustic waves from a point source and received at microphones $i$ and $j$.

$$
\begin{aligned}
& p_{a_{i}}(t)=\frac{A}{R_{a_{i}}} \exp \left(i k R_{a_{i}}\right) \sin \left(2 \pi f_{0} t\right) \\
& p_{a_{j}}(t)=\frac{A}{R_{a_{j}}} \exp \left(i k R_{a_{j}}\right) \sin \left(2 \pi f_{0} t\right)
\end{aligned}
$$

where $R_{a_{i}}$ is the distance from the point source $a$ to microphone $i$, and $R_{a_{j}}$ is the distance from the point source $a$ to microphone $j$.

For the one-sided autospectral density and the one-sided crossspectral density, we have

$$
\begin{gathered}
G_{p_{a_{i}} p_{a_{i}}}(f)=\frac{1}{2} \frac{A^{2}}{R_{a_{i}}^{2}} \delta\left(f-f_{0}\right) \\
G_{p_{a_{i} p_{a_{j}}}}(f)=\frac{1}{2} \frac{A^{2}}{R_{a_{i}} R_{a_{j}}} e^{-j k\left(R_{a_{i}}-R_{a_{j}}\right)} \delta\left(f-f_{0}\right)
\end{gathered}
$$

\section{Appendix B: Correlated Noise Source $A$}

Consider the $i$ th and $j$ th microphones and a single acoustic point source. As shown in Appendix A, the autospectra are

$$
\begin{aligned}
G_{p_{a_{i}} p_{a_{i}}}(f) & =\frac{1}{2} \frac{A^{2}}{R_{a_{i}}^{2}} \delta\left(f-f_{0}\right) \\
G_{p_{a_{j} p_{a_{j}}}}(f) & =\frac{1}{2} \frac{A^{2}}{R_{a_{j}}^{2}} \delta\left(f-f_{0}\right)
\end{aligned}
$$

and the cross spectrum is

$$
G_{p_{a_{i}} p_{a_{j}}}(f)=\frac{1}{2} \frac{A^{2}}{R_{a_{i}} R_{a_{j}}} e^{-j k\left(R_{a_{i}}-R_{a_{j}}\right)} \delta\left(f-f_{0}\right)
$$

\section{Appendix C: Correlated Noise Source $A$ and Uncorrelated Source $N$}

Consider the $i$ th and $j$ th microphones, a single acoustic point source $A$, and a noise source $N$. As shown in Appendix $\underline{\mathrm{A}}$, the autospectra are

$$
\begin{aligned}
& G_{p_{a_{i}} p_{a_{i}}}(f)=\frac{1}{2} \frac{A^{2}}{R_{a_{i}}^{2}} \delta\left(f-f_{0}\right)+\frac{1}{2} \frac{N^{2}}{R_{n_{i}}^{2}} \delta\left(f-f_{0}\right) \\
& G_{p_{a_{j}} p_{a_{j}}}(f)=\frac{1}{2} \frac{A^{2}}{R_{a_{j}}^{2}} \delta\left(f-f_{0}\right)+\frac{1}{2} \frac{N^{2}}{R_{n_{j}}^{2}} \delta\left(f-f_{0}\right)
\end{aligned}
$$

and the cross spectrum is

$$
G_{p_{a_{i}} p_{a_{j}}}(f)=\frac{1}{2} \frac{A^{2}}{R_{a_{i}} R_{a_{j}}} e^{-j k\left(R_{a_{i}}-R_{a_{j}}\right)} \delta\left(f-f_{0}\right)
$$

\section{Appendix D: Correlated Noise Source $A$ and Correlated Noise Source $B$}

Consider the $i$ th and $j$ th microphones, a point acoustic source $A$ and a point acoustic source $B$. As shown in Appendix $\underline{A}$, the autospectra are

$$
\begin{aligned}
& G_{p_{a_{i}} p_{a_{i}}}(f)=\frac{1}{2} \frac{A^{2}}{R_{a_{i}}^{2}} \delta\left(f-f_{0}\right)+\frac{1}{2} \frac{B^{2}}{R_{b_{i}}^{2}} \delta\left(f-f_{0}\right) \\
& G_{p_{a_{j}} p_{a_{j}}}(f)=\frac{1}{2} \frac{A^{2}}{R_{a_{j}}^{2}} \delta\left(f-f_{0}\right)+\frac{1}{2} \frac{B^{2}}{R_{b_{j}}^{2}} \delta\left(f-f_{0}\right)
\end{aligned}
$$

and the cross spectrum is

$$
\begin{gathered}
G_{p_{i} p_{j}}(f)=\frac{1}{2} \frac{A^{2}}{R_{a_{i}} R_{a_{j}}} e^{-j k\left(R_{a_{i}}-R_{a_{j}}\right)} \delta\left(f-f_{0}\right) \\
+\frac{1}{2} \frac{B^{2}}{R_{b_{i}} R_{b_{j}}} e^{-j k\left(R_{b_{i}}-R_{b_{j}}\right)} \delta\left(f-f_{0}\right)
\end{gathered}
$$

\section{Appendix E: Correlated Noise Source $A$, Correlated Noise Source $B$, and Uncorrelated Source $N$}

Consider the $i$ th and $j$ th microphones, a single acoustic point source $A$, and a noise source $N$. As shown in Appendix $\underline{\mathrm{A}}$, the autospectra are

$$
G_{p_{a_{i}} p_{a_{i}}}(f)=\frac{1}{2} \frac{A^{2}}{R_{a_{i}}^{2}} \delta\left(f-f_{0}\right)+\frac{1}{2} \frac{B^{2}}{R_{b_{i}}^{2}} \delta\left(f-f_{0}\right)+\frac{1}{2} \frac{N^{2}}{R_{n_{i}}^{2}} \delta\left(f-f_{0}\right)
$$

$$
G_{p_{a_{j}} p_{a_{j}}}(f)=\frac{1}{2} \frac{A^{2}}{R_{a_{j}}^{2}} \delta\left(f-f_{0}\right)+\frac{1}{2} \frac{B^{2}}{R_{b_{j}}^{2}} \delta\left(f-f_{0}\right)+\frac{1}{2} \frac{N^{2}}{R_{n_{j}}^{2}} \delta\left(f-f_{0}\right)
$$

and the cross spectrum is

$$
\begin{gathered}
G_{p_{i} p_{j}}(f)=\frac{1}{2} \frac{A^{2}}{R_{a_{i}} R_{a_{j}}} e^{-j k\left(R_{a_{i}}-R_{a_{j}}\right)} \delta\left(f-f_{0}\right) \\
+\frac{1}{2} \frac{B^{2}}{R_{b_{i}} R_{b_{j}}} e^{-j k\left(R_{b_{i}}-R_{b_{j}}\right)} \delta\left(f-f_{0}\right)
\end{gathered}
$$

\section{Appendix F: Cost Function}

The cost function described is for the third model, which is the five-parameter case $\left[\operatorname{SPL}_{N}(f), \operatorname{SPL}_{A}(f), \operatorname{SPL}_{B}(f), x_{A}(f)\right.$, and $\left.x_{B}(f)\right]$. Measured values of the autospectra $\operatorname{splayy}(f, \ell+2, \ell+2)$, cross-spectra magnitude splayy $(f, \ell+2, m+2)$, cross-spectra 
Table F1 Cost-function angle relationships

\begin{tabular}{ccc}
\hline \hline Microphone identification & $\theta_{\text {id }}$, deg & $\theta_{\text {model }}$, deg \\
\hline 1 & 100 & 280 \\
2 & 110 & 290 \\
3 & 120 & 300 \\
4 & 130 & 310 \\
\hline \hline
\end{tabular}

phase angle $\operatorname{phay}(f, \ell+2, m+2)$, and coherence $\gamma^{2}(f, \ell+$ $2, m+2)$ are assumed to be available. The cost function is minimized at each frequency.

The third model in the sequence assumes that the data are the result of a source triplet consisting of a coherent source at location $x_{A}(f)$ with $y_{A}=0.0$ of strength $\operatorname{SPL}_{A}(f)$ (identified with index 5), a coherent source at location $x_{B}(f)$ with $y_{B}=0.0$ of strength $\mathrm{SPL}_{B}(f)$ (identified with index 6), and an incoherent source of strength $\operatorname{SPL}_{N}(f)$ at the location $x_{N}=0.0$ and $y_{N}=0.0$ (identified with index 7).

The far-field microphone model angles used in the cost function are shown in Table F1 and indices 1 through 4 are used. The pressure parameters are transformed to pressures using

$$
\begin{gathered}
p_{A}=A=r_{0} p_{o} 10^{\mathrm{SPL}_{A} / 20} \quad p_{B}=B=r_{0} p_{o} 10^{\mathrm{SPL}_{B} / 20} \\
p_{N}=N=r_{0} p_{o} 10^{\mathrm{SPL}_{N} / 20}
\end{gathered}
$$

The locations of the far-field microphones and sources are given by

$$
\begin{array}{ccc}
x g(\ell)=r_{0} 150 \cos \left[\theta_{\text {model }}(\ell) \pi / 180\right] & \ell=1,2,3,4 \\
y g(\ell)=r_{0} 150 \sin \left[\theta_{\text {model }}(\ell) \pi / 180\right] & \ell=1,2,3,4 \\
x g(5)=r_{0} x_{A} & y g(5)=0.0 & x g(6)=r_{0} x_{B} \\
y g(6)=0.0 & x g(7)=0.0 & y g(7)=0.0
\end{array}
$$

The separation distance between the sources and the far-field microphones are given by

$$
\begin{aligned}
& r(5, m)=\sqrt{[x g(5)-x g(m)]^{2}+[y g(5)-y g(m)]^{2}} \\
& m=1,2,3,4 \\
& r(6, m)=\sqrt{[x g(6)-x g(m)]^{2}+[y g(6)-y g(m)]^{2}} \\
& m=1,2,3,4 \\
& r(7, m)=\sqrt{[x g(7)-x g(m)]^{2}+[y g(7)-y g(m)]^{2}} \\
& m=1,2,3,4
\end{aligned}
$$

The autospectra components are calculated as

$$
\begin{gathered}
\operatorname{tg} u(\ell, \ell)=0.5 p_{A}^{2} /\left[r(5, \ell)^{2}\right] \quad \operatorname{tnu}(\ell, \ell)=0.5 p_{B}^{2} /\left[r(6, \ell)^{2}\right] \\
\operatorname{tgnn}(\ell, \ell)=0.5 p_{N}^{2} /\left[r(7, \ell)^{2}\right]
\end{gathered}
$$

where $\ell=1,2,3,4$.

The cross-spectra phase is calculated using

$$
\begin{aligned}
& \phi_{\alpha}(\ell, m)=-k[r(5, \ell)-r(5, m)] \\
& \phi_{\beta}(\ell, m)=-k[r(6, \ell)-r(6, m)]
\end{aligned}
$$

where $\ell$ goes from 1 to 3 , and for each $\ell$ value, $m$ goes from $\ell+1$ to 4 and the wave number $k$ is $2 \pi f / c_{o}$.

The cross-spectra phase components are

$$
\begin{aligned}
& \operatorname{tg} u(\ell, m)=0.5\left\{\exp \left[j \phi_{\alpha}(\ell, m)\right]\right\} p_{A}^{2} /[r(5, \ell) r(5, m)] \\
& \operatorname{tnu}(\ell, m)=0.5\left\{\exp \left[j \phi_{\beta}(\ell, m)\right]\right\} p_{B}^{2} /[r(6, \ell) r(6, m)]
\end{aligned}
$$

where $\ell$ goes from 1 to 3 , and for each $\ell$ value, $m$ goes from $\ell+1$ to 4.
The autospectra at each microphone are calculated using

$$
\begin{gathered}
\operatorname{tg} y(\ell, \ell)=\operatorname{tg} u(\ell, \ell)+\operatorname{tgn} u(\ell, \ell)+\operatorname{tgnn}(\ell, \ell) \\
\operatorname{tsply}(\ell, \ell)=10.0 \log _{10}\left[\operatorname{tg} y(\ell, \ell) / p_{0}^{2}\right]
\end{gathered}
$$

where $\ell$ goes from 1 to 4 .

The autospectra error cost function sum 1 is then

$$
\operatorname{sum} 1=\frac{25.0}{\mathrm{~dB}^{2}} \sum_{\ell=1}^{4}[\operatorname{tsply}(\ell, \ell)-\operatorname{splayy}(f, \ell+2, \ell+2)]^{2}
$$

The cross-spectra magnitude and phase at each microphone are calculated using

$$
\begin{gathered}
\operatorname{tg} y(\ell, m)=\operatorname{tgu}(\ell, m)+\operatorname{tgnu}(\ell, m) \\
\operatorname{tsply}(\ell, m)=10.0 \log _{10}\left[\operatorname{tg} y(\ell, m) / p_{0}^{2}\right] \\
\operatorname{tphay}(\ell, m)=\arctan \{\Im[\operatorname{tg} y(\ell, m)], \Re[\operatorname{tg} y(\ell, m)]\} * 180 / \pi
\end{gathered}
$$

where $\ell$ goes from 1 to 3 , and for each $\ell$ value, $m$ goes from $\ell+1$ to 4.

The cross-spectra magnitude error cost function sum 2 is then

$$
\operatorname{sum} 2=\left(\frac{1}{6 \mathrm{~dB}^{2}}\right) \sum[\operatorname{tsply}(\ell, m)-\operatorname{splayy}(f, \ell+2, m+2)]^{2}
$$

where in doing the summation, $\ell$ goes from 1 to 3 , and for each $\ell$ value, $m$ goes from $\ell+1$ to 4 .

In calculating the cross-spectrum phase-angle cost function, we first calculate a normalizing weighting factor from the measured coherence values:

$$
\text { sumgam }=\sum \gamma^{2}(f, \ell+2, m+2)
$$

where in doing the summation, $\ell$ goes from 1 to 3 , and for each $\ell$ value, $m$ goes from $\ell+1$ to 4 .

Next, we calculate a phase-angle error based on the distance between points on a unit circle weighted by the measure coherence:

$$
\begin{gathered}
\operatorname{angx}(\ell, m)=\cos [\operatorname{tphay}(\ell, m) \times \pi / 180] \\
\operatorname{angy}(\ell, m)=\sin [\operatorname{tphay}(\ell, m) \times \pi / 180] \\
\operatorname{angx} 2(\ell, m)=\cos [\operatorname{phay}(f, \ell+2, m+2) \times \pi / 180] \\
\operatorname{angy2}(\ell, m)=\sin [\operatorname{phay}(f, \ell+2, m+2) \times \pi / 180] \\
\operatorname{angrad} 2(\ell, m)=[\operatorname{angx}(\ell, m)-\operatorname{angx} 2(\ell, m)]^{2} \\
+[\operatorname{angy}(\ell, m)-\operatorname{angy} 2(\ell, m)]^{2} \\
\operatorname{phawt}(l, m)=\gamma^{2}(f, \ell+2, m+2) \operatorname{angrad} 2(\ell, m) / \operatorname{sumgam}
\end{gathered}
$$

The cross-spectra phase-angle-error cost function sum $3 \mathrm{wt}$ is then

$$
\operatorname{sum} 3 \mathrm{wt}=1000 \sum \operatorname{phawt}(l, m)
$$

where in doing the summation, $\ell$ goes from 1 to 3 , and for each $\ell$ value, $m$ goes from $\ell+1$ to 4 .

The total cost function is

$$
\begin{aligned}
& \operatorname{sumf}=\operatorname{sum} 1+\operatorname{sum} 2+\operatorname{sum} 3 w t+\operatorname{con} p 2 n 50 \\
& +w t 1+w t 2+w t 3
\end{aligned}
$$




$$
\begin{aligned}
& \text { conp } 2 n 50=\left\{\begin{array}{l}
0: \\
\frac{1}{\mathrm{~dB}}\left(10-\mathrm{SPL}_{N}\right)^{2}: \quad \text { if } \mathrm{SPL}_{N}<10
\end{array}\right. \\
& x \text { hhi } A=\left\{\begin{array}{l}
100.0 \\
25.0
\end{array} \text { if } f>164 \quad x 5 \operatorname{low} A=11.0\right. \\
& x 5 \operatorname{hi} B=\left\{\begin{array}{l}
100.0 \\
11.0
\end{array} \text { if } f>164 \quad x 5 \operatorname{low} B=-25.0\right. \\
& w t 1= \begin{cases}0: & \\
\frac{100}{\mathrm{ft}^{2}}\left(x_{A}-x 5 \mathrm{hi}\right)^{2}: & \text { if } x_{A}>x 5 \mathrm{hi} \\
\frac{100}{\mathrm{ft}^{2}}\left(x_{A}-x 5 \mathrm{low}\right)^{2}: & \text { if } x_{A}<x 5 \text { low }\end{cases} \\
& w t 2= \begin{cases}0: & \\
\frac{100}{\mathrm{ft}^{2}}\left(x_{B}-x 5 \mathrm{hi} B\right)^{2}: & \text { if } x_{B}>x 5 \mathrm{hi} B \\
\frac{100}{\mathrm{ft}^{2}}\left(x_{B}-x 5 \operatorname{low} B\right)^{2}: & \text { if } x_{B}<x 5 \operatorname{low} B\end{cases} \\
& w t 3=\left\{\begin{array}{l}
0: \\
\frac{100}{\mathrm{ft}^{2}}\left(x_{B}-x_{A}\right)^{2}: \text { if } x_{A}>x_{B}
\end{array}\right.
\end{aligned}
$$

The functions conp2n50, wt 1 , and $w t 2$ are used to keep the parameters within bounds. For $f<164$, we have $11<x_{A}<100$ and $-25<x_{B}<100$, and for $f>164$, we have $11<x_{A}<25$ and $-25<x_{B}<11$. The $w t 3$ function is used to keep point source $A$ separate from point source $B$. It removes the symmetry in the system model. The symmetry permits the interchange of the parameters associated with the coherent sources $A$ and $B$ without any change in the error. Rather than sorting the answers after the solution is obtained, the $w t 3$ cost function forces the desired result.

\section{References}

[1] Miles, J. H., "Aligned and Unaligned Coherence: A New Diagnostic Tool, AIAA Paper 2006-0010, 2006; also NASA TM-2006-214112, Jan. 2006

[2] Miles, J. H., "Validating Coherence Measurements Using Aligned and Unaligned Coherence Functions," AIAA Paper 2006-1389, 2006; also NASA TM-2006-214113, Jan. 2006.

[3] Miles, J. H., "Restricted Modal Analysis Applied to Internal Annular Combustor Autospectra and Cross-Spectra Measurements," AIAA Journal, Vol. 45, No. 5, May 2007, pp. 988-999. doi:10.2514/1.25179

[4] Bendat, J. S., and Piersol, A. G., Measurement and Analysis of Random Data, Wiley, New York, 1966.

[5] Bendat, J. S., and Piersol, A. G., Random Data: Analysis and Measurement Procedures, Wiley, New York, 1971.

[6] Bendat, J. S., and Piersol, A. G., Engineering Applications of Correlation and Spectral Analysis, Wiley, New York, 1980.

[7] Karchmer, A. M., "Identification And Measurement of Combustion Noise from a Turbofan Engine Using Correlation and Coherence Techniques," NASA TM-73747, 1977.

[8] Karchmer, A. M., Reshotko, M., and Montegani, F. J., "Measurement of Far Field Combustion Noise from a Turbofan Engine Using Coherence Functions," AIAA Paper 77-1277, 1977; also NASA TM73748, Oct. 1977.

[9] Chung, J. Y., Crocker, M. J., and Hamilton, J. F. , "Measurement of Frequency Response and the Multiple Coherence Function of the Noise-Generation System of a Diesel Engine," Journal of the Acoustical Society of America, Vol. 58, No. 3, Sept. 1975, pp. 635-642. doi:10.1121/1.380703

[10] Chung, J. Y., "Rejection of Flow Noise Using a Coherence Function Method," Journal of the Acoustical Society of America, Vol. 62, No. 2, Aug. 1977, pp. 388-395. doi:10.1121/1.381537

[11] Krejsa, E. A., "New Technique for the Direct Measurement of Core Noise from Aircraft Engines," NASA TM-82634, 1981.
[12] Shivashankara, B. N., "High Bypass Ratio Engine Noise Component Separation by Coherence Technique," Journal of Aircraft, Vol. 20, No. 3, Mar. 1983, pp. 236-242; AIAA Paper 1981-2054, Oct. 1981.

[13] Hsu, J. S., and Ahuja, K. K., "A Coherence-Based Technique to Separate Ejector Internal Mixing Noise from Farfield Measurements," AIAA Paper 98-2296, June 1998.

[14] Stoker, R. W., Ahuja, K. K., and Hsu, J., "Separation of Wind-Tunnel Background Noise and Wind Noise from Automobile Interior Measurements," AIAA Paper 96-1763, May 1996.

[15] Michalke, A., Arnold, F., and Holste, F., "On the Coherence of the Sound Field in a Circular Duct with Uniform Mean Flow," Journal of Sound and Vibration, Vol. 190, No. 2, 1996, pp. 261-271. doi:10.1006/jsvi.1996.0061

[16] Minami, T., and Ahuja, K. K., "Five-Microphone Method for Separating Two Different Correlated Noise Sources from Far Field Measurements Contaminated by Extraneous Noise," AIAA Paper 20033261, May 2003.

[17] Parthasarathy, S. P., Cuffel, R. F., and Massier, P. F., "Separation of Core Noise and Jet Noise," AIAA Journal, Vol. 18, No. 3, 1980, pp. 256-261; also AIAA Paper 1979-589, 1979.

[18] Tesson, V., "Experimental Investigation of Jet Noise and Core Noise Using a Small Gas Turbine Engine," M.S. Thesis, Dept. of Aerospace Engineering, Pennsylvania State Univ., State College, PA, Aug. 2002.

[19] Tesson, V., "Experimental Investigation of Jet Noise and Core Noise Using a Small Gas Turbine Engine," AIAA Paper 2002-2558, June 2002

[20] Dougherty, R. P.," Beamforming in Acoustic Testing, Aeroacoustic Measurements, edited by T. J. Mueller, Springer-Verlag, Berlin, 2002, pp. 62-97, Chap. 2.

[21] Underbrink, J. R., "Aeroacoustic Phased Array Testing in Low Speed Wind Tunnels," Aeroacoustic Measurements, edited by T. J. Mueller, Springer-Verlag, Berlin, 2002, pp. 98-217, Chap. 2.

[22] Siller, H. A., Arnold, F., and Michel, U., "Investigation of Aero-Engine Core-Noise Using a Phased Micro-Phone Array," 7th AIAA/CEAS Aeroacoustics Conference, AIAA Paper 2001-2269, 2001.

[23] Oerlemans, S., and Sijtsma, P., "Determination of Absolute Levels from Phased Array Measurements Using Spatial Source Coherence," 8th AIAA/CEAS Aeroacoustics Conference, AIAA Paper 2002-2464, 2002.

[24] Lee, S. S., and Bridges, J., "Phased-Array Measurements of Single Flow Hot Jets," 11th AIAA/CEAS Aeroacoustics Conference, AIAA Paper 2005-2842, 2005.

[25] Suzuki, T., "Coherent Noise Radiation from a Jet Investigated with a Beam-Forming Technique," 12th AIAA/CEAS Aeroacoustics Conference, AIAA Paper 2006-2646, 2006.

[26] Lee, S. S., "Phased-Array Measurements of Modern Regional Aircraft Turbofan Engine Noise," 12th AIAA/CEAS Aeroacoustics Conference, AIAA Paper 2006-2653, 2006.

[27] Flynn, O. E., and Kinns, R., "Multiplicative Signal Processing for Sound Source Location on Jet Engines," Journal of Sound and Vibration, Vol. 46, No. 1, 1976, pp. 137-150. doi:10.1016/0022-460X(76)90823-3

[28] Billingsley, J., and Kinns, R., "The Acoustic Telescope," Journal of Sound and Vibration, Vol. 48, No. 4, 1976, pp. 485-510. doi:10.1016/0022-460X(76)90552-6

[29] Fisher, M. J., Harper-Bourne, M., and Glegg, S. A. L., "Jet Engine Noise Source Location: The Polar Correlation Technique," Journal of Sound and Vibration, Vol. 51, No. 1, 1977, pp. 23-54. doi:10.1016/S0022-460X(77)80111-9

[30] Blake, W. K., and Lynch, D. A., "Source Characterization by Correlation Techniques," Aeroacoustic Measurements, edited by T. J. Mueller, Springer-Verlag, Berlin, 2002, pp. 218-257, Chap. 2.

[31] Kinns, R., "Binaural Source Location," Journal of Sound and Vibration, Vol. 44, No. 2, 1976, pp. 275-289. doi:10.1016/0022-460X(76)90774-4

[32] Williams, F., "Anti-Sound," Modern Methods in Analytical Acoustics, edited by D. G. Crighton, Springer-Verlag, Berlin, 1992, Chap. 26.

[33] Brent, R. P., Algorithms for Minimization Without Derivatives, Prentice-Hall, Englewood Cliffs, NJ, 2002.

[34] Powell, M. J. D., "An Efficient Method of Finding the Minimum of a Function of Several Variables Without Calculating Derivatives," Computer Journal, Vol. 7, No. 2, July 1964, pp. 155-162. doi:10.1093/comjnl/7.2.155

[35] Shapiro, M. S., and Goldstein, M., "A Collection of Mathematical Computer Routines," New York Univ., AEC Computing and Applied Mathematics Center, Rept. NYO-1480-14, New York, Feb. 1965.

[36] Kuester, J. L., and Mize, J. H., "Optimization Techniques with Fortran," McGraw-Hill, New York, 1973. 
[37] Stearns, S. D., and David, R. A., "Signal Processing Algorithms Using Fortran and C.," Prentice-Hall, Englewood Cliffs, NJ, 1993.

[38] Carter, G. C., "Receiver Operating Characteristics for a Linearly Thresholded Coherence Estimation Detector," IEEE Transactions on Acoustics, Speech, and Signal Processing, Vol. 25, No. 1, Feb. 1977, pp. 90-92. doi:10.1109/TASSP.1977.1162898

[39] Carter, G. C., "Coherence and Time Delay Estimation," Proceedings of the IEEE, Vol. 75, No. 2, Feb. 1987, pp. 236-255.

[40] Halliday, D. M., Rosenberg, J. R., Amjad, A. M., Breeze, P., Conway, B. A., and Farmer, S. F., "A Framework for the Analysis of Mixed Time Series/Point Process Data-Theory and Application to the Study of Physiological Tremor," Progress in Biophysics and Molecular Biology, Vol. 64, No. 2, 1995, pp. 237-278. doi:10.1016/S0079-6107(96)00009-0
[41] Brillinger, D. R., Time Series: Data Analysis and Theory, Holden-Day, San Francisco, 1981. ISBN:0-8162-1150-7.

[42] Piersol, A., "Time Delay Estimation Using Phase Data," IEEE Transactions on Acoustics, Speech, and Signal Processing, Vol. 29, No. 3, June 1981, pp. 471-477. doi:10.1109/TASSP.1981.1163555

[43] Rose, C., and Smith, M. D., Mathematical Statistics with Mathematica, Springer-Verlag, New York, 2002, p. 181.

[44] Shivashankara, B. N., "Aircraft Noise Prediction Program Validation," NASA CR-159333, Oct. 1980.

[45] Morse, P. M., and Ingard, K. U., Theoretical Acoustics, McGraw-Hill, New York, 1968. 